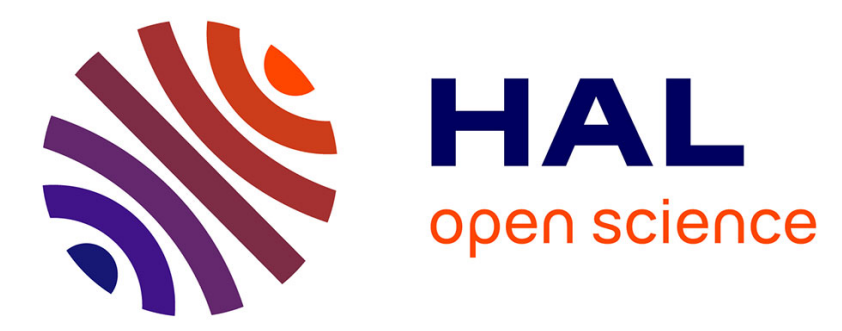

\title{
Effective electrical conductivity of transversely isotropic rocks with arbitrarily oriented ellipsoidal inclusions
}

\author{
A. Giraud, I. Sevostianov, I. Kushch, P. Cosenza, D. Pret, Jean-François \\ Barthélémy, A. Trofimov
}

\section{- To cite this version:}

A. Giraud, I. Sevostianov, I. Kushch, P. Cosenza, D. Pret, et al.. Effective electrical conductivity of transversely isotropic rocks with arbitrarily oriented ellipsoidal inclusions. Mechanics of Materials, 2019, 10.1016/j.mechmat.2019.03.011 . hal-02075389

\section{HAL Id: hal-02075389 \\ https://hal.science/hal-02075389}

Submitted on 22 Oct 2021

HAL is a multi-disciplinary open access archive for the deposit and dissemination of scientific research documents, whether they are published or not. The documents may come from teaching and research institutions in France or abroad, or from public or private research centers.
L'archive ouverte pluridisciplinaire HAL, est destinée au dépôt et à la diffusion de documents scientifiques de niveau recherche, publiés ou non, émanant des établissements d'enseignement et de recherche français ou étrangers, des laboratoires publics ou privés.

\section{(ㄷ)(1) $\$$}

Distributed under a Creative Commons Attribution - NonCommerciall 4.0 International 


\title{
Effective electrical conductivity of transversely isotropic rocks with arbitrarily oriented ellipsoidal inclusions
}

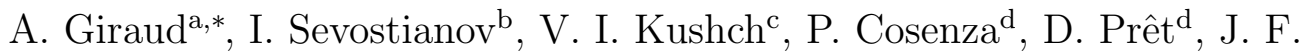 \\ Barthélémy $^{\mathrm{e}}$, A. Trofimov ${ }^{\mathrm{f}}$ \\ ${ }^{a}$ GeoRessources Laboratory, Université de Lorraine (ENSG), CNRS, CREGU, F-54518 \\ Vandoeuvre-lès-Nancy, France \\ ${ }^{b}$ Department of Mechanical and Aerospace Engineering, New Mexico State University, \\ Las Cruces, NM 88001, USA \\ ${ }^{c}$ Institute for Superhard Materials of the National Academy of Sciences, 04074 Kiev, \\ Ukraine \\ ${ }^{d} I C 2 M P-H y d r A S A$, Université de Poitiers, CNRS, UMR 7285, Poitiers, France \\ ${ }^{e}$ Cerema, Project-team DIMA, 110 rue de Paris, BP 214, 77487 Provins Cedex, France \\ ${ }^{f}$ Department of Mechanical Engineering, Polytechnique Montreal, Canada
}

\begin{abstract}
This paper addresses the problem of the electrical conductivity tensor calculation for a transversely isotropic material that contains inhomogeneities of arbitrary orientation. For this goal, we first construct the electrical conductivity contribution tensor for an arbitrarily oriented isolated ellipsoidal anisotropic inhomogeneity embedded in a transversely isotropic matrix. The general case of an orthotropic ellipsoidal inhomogeneity unaligned in an anisotropic matrix with different classes of symmetry can be considered. This solution is used as the basic building block of various homogenization techniques: the Mori-Tanaka-Benveniste scheme, Maxwell scheme, and differential scheme. The approach is illustrated by an application to a transversely isotropic mudstone rock, composed of a clay matrix containing inhomogeneities of calcite and quartz. We analyse the origins of the extent of anisotropy of the effective conductivity tensor, distinguishing among the

\footnotetext{
${ }^{*}$ Corresponding author

Email addresses: albert.giraud@univ-lorraine.fr (A. Giraud), igor.sevostianov@nmsu.edu (I. Sevostianov), vkushch@bigmir.net (V. I. Kushch), philippe.cosenza@univ-poitiers.fr (P. Cosenza), dimitri.pret@univ-poitiers.fr (D. Prêt), jf.barthelemy@cerema.fr (J. F. Barthélémy), anton.trofimov@polymtl.ca (A. Trofimov)
} 
shape, orientation distribution, and anisotropy of the inhomogeneities on the one hand and the anisotropy of the matrix on the other hand. Numerical results show that the orientation distribution of the inhomogeneities significantly affects the overall anisotropy in the case of inhomogeneities with low aspect ratio(s). Limiting cases of aligned and randomly oriented inhomogeneities provide bounds of the extent of anisotropy for the overall conductivity tensor.

Keywords: effective electrical conductivity, arbitrarily oriented inclusion, ellipsoidal inclusion, transversely isotropic rock

\section{Introduction}

The present paper is motivated mostly by the needs of rock physics, although it is relevant to many other applications for which heterogeneous materials with anisotropic constituents are studied. Clay rocks, or argillaceous rocks, are cap rocks of oil and gas reservoirs and are presently used in many engineering applications, such as underground works (e.g., mines and quarries) and storage cavities (e.g., high-level radioactive waste repositories and storage of $\mathrm{CO}_{2}, \mathrm{H}_{2}$, etc.).

The microstructure of these materials has been extensively studied during the last decades, and many multiscale models have been proposed; see, among others, $[1,2,3]$. As an example, four microstructural levels have been proposed by $[2,4]$, from the scale of elementary clay minerals (level 0, nanometer scale) to the macroscopic scale (level 3, figure 8). At the mesoscale (micrometer to millimeter scale, corresponding to level 2 in figure 8), clay-rich sedimentary rocks can be considered as a particulate matrix composite type, with a porous clay matrix containing solid mineral inhomogeneities [5]. The clay matrix, in turn, is also heterogeneous, composed of a mixture of different clay minerals, and inhomogeneities are represented by various solid minerals

(as carbonates and quartz) and pores. These different solid mineral phases will be referred to as non-clay phases in the following.

The effects of the microscopic organization of non-clay inhomogeneities, on the one hand, and the microscopic organization of the clay matrix, on the other hand, on the effective transport properties of clay rocks have been investigated both theoretically and experimentally; see the review in [6]. In the case of Callovo-Oxfordian mudstone from the Paris sedimentary basin, extensively studied in France as a potential host rock for high-level radioactive 
waste repositories, petrographic observations [5] have shown that carbonates and quartz inhomogeneities may have a preferential orientation parallel to the bedding plane, and elongated shapes have been observed for carbonates. [7] presented similar results for the Opalinus clay rock unit at the Mont Terri rock laboratory (Canton Jura, Switzerland) and analysed the impact on the pore path tortuosity and then on the transfer properties. These studies have confirmed that non-clay inclusion distributions (orientation, shape, and spatial organization) may be an origin of the anisotropy of the effective transport property, especially electrical conductivity.

The main source of the effective anisotropy of transport properties may also lie in the microstructural organization of the clay matrix phase, which contains clay minerals and pores. Most of the previous studies have only focused on the clay matrix anisotropy. In the particular case of electrical conductivity, the reader may refer to the series of papers $[8,9,10]$ for a review of cross-coupled modelling of the flow of electric charges, ions, and water in the clay matrix, including its homogenization, accounting for filtration and diffusion effects. A review and experimental data have been presented by [11] for the effective electrical conductivity of anisotropic mudstones. The major source of anisotropy of the effective electrical conductivity tensor of argillaceous rocks, identified by most authors, is the preferential alignment of pores and clay particles (platelets) in the clay matrix $[12,11]$.

The key problem of homogenization of the clay matrix phase from the nanoscale to the mesoscale is not investigated in this paper, which is devoted to the transition from the mesoscale to the macroscale (transition from level 2 to level 3, figure 8). The clay matrix phase will be considered as a homogeneous anisotropic material. As the quantitative connection of the organization of non-clay inclusions to macroscopic electrical conductivity is still a critical issue in the case of matrix anisotropy, we focused the paper on this point.

Most of these clay-rich sedimentary rocks are transversely isotropic, and we will restrain the study to this class of anisotropy. The main objective of this paper is to contribute to identifying the microstructural origin of the anisotropy of the effective electrical conductivity tensor, accounting for the available microstructural information. The key problem is to compare effects produced by the two main sources: the matrix anisotropy and anisotropy of the inhomogeneities (including their properties, shape, and orientation distribution). The three main approaches to calculate the effective electrical conductivity tensor of anisotropic heterogeneous materials are (1) semi-empirical approaches mainly based on Archie's relationship, (2) numerical models, and 
(3) analytical homogenization techniques (see, for example, [6] for details). In this paper, we focus on the third approach based on the conductivity problem for an isolated ellipsoidal inhomogeneity embedded in an anisotropic medium obtained independently by [13] and [14], in which an analytical expression of the Hill conductivity polarization tensor is obtained from the known solution of the Newtonian potential of an ellipsoid using a linear coordinate transform. This analytical solution can be used to construct the property contribution tensors of an arbitrarily oriented ellipsoidal inhomogeneity embedded in a transversely isotropic matrix and to analytically estimate the overall properties of an anisotropic material. The latter is necessary to evaluate the effects of the two abovementioned sources of the overall anisotropy - the matrix anisotropy and anisotropy associated with the geometry and properties of the non-clay inhomogeneities.

In the context of anisotropic materials and, in particular, the transversely isotropic rocks investigated in this paper, this analytical solution of the Hill conductivity tensor allows consideration of the arbitrary orientation of inclusions, and it provides the possibility of separating the two previously cited sources of effective anisotropy, the matrix anisotropy and geometric anisotropy associated with inclusions phases. In particular, this solution has been applied to calculate the effective permeability of multifractured rocks (see $[13,15])$. In the context of electrical conductivity, to the best of our knowledge, only aligned inhomogeneities in an anisotropic matrix have been studied. In the case of the thermal conductivity tensor, the case of arbitrarily oriented inclusions in a transversely isotropic matrix with application to a mudstone has been discussed by [16] using a numerical solution for the conductivity Hill tensor. An analytical solution application is presented for the effective electrical conductivity of a transversely isotropic clay rock, CallovoOxfordian mudstone from the Paris sedimentary basin, which is extensively studied in France as a potential host rock for high-level radioactive waste repositories (see [17]). The most popular homogenization techniques, such as the Mori-Tanaka-Benveniste (MTB) scheme, Maxwell scheme, and differential scheme $(D S)$, are compared for the problem of inversion of the matrix electrical conductivity tensor obtained from effective experimental data, accounting for microstructural information. 


\section{Effective medium approximations of the effective electrical con- ductivity tensor}

\subsection{Notations for the conductivity problem}

Bold symbols $\boldsymbol{P}, \boldsymbol{\sigma}$, and $\boldsymbol{i}$ refer to second-order tensors, underlined symbols, such as $\underline{a}$ refer to first-order tensors. $\boldsymbol{i}$ represents the second-order identity tensor. $\boldsymbol{\sigma}$ denotes the second-order electrical conductivity tensor $[12,6]$.

\subsection{Mori-Tanaka-Benveniste and Maxwell approximations}

The property contribution tensor approach initially formulated in elasticity [18] and in conductivity [19] will be adopted, as it allows a simple and direct way to extend the mean field homogenization methods to complex inclusion shapes. See [20] for applications to electrical conductivity. For the sake of simplicity, we only recall the formula in the case of of inclusions of the same shape, the same conductive properties, and volume fraction $\varphi_{i}$. The reference dilute approximation in a conductivity formulation is useful in the low limit of the volume fraction of inclusions $\varphi_{i} \ll 1$, written as

$$
\boldsymbol{\sigma}^{\mathrm{DIL}}=\boldsymbol{\sigma}_{0}+\varphi_{i}\left(\boldsymbol{\sigma}_{i}-\boldsymbol{\sigma}_{0}\right) \cdot \boldsymbol{A}_{i}
$$

with the dilute conductivity concentration tensor

$$
\boldsymbol{A}_{i}=\left(\boldsymbol{i}+\boldsymbol{P}_{i}^{0} \cdot\left(\boldsymbol{\sigma}_{i}-\boldsymbol{\sigma}_{0}\right)\right)^{-1}
$$

$\boldsymbol{P}_{i}^{0}$ denotes the Hill tensor of a single ellipsoidal inhomogeneity $i$ embedded in an infinite homogeneous matrix with conductivity tensor $\boldsymbol{\sigma}_{0}$ (see section 6). Relation (39) may be rewritten using the conductivity contribution tensor $N_{i}$

$$
\boldsymbol{\sigma}^{\mathrm{DIL}}=\boldsymbol{\sigma}_{0}+\varphi_{i} \boldsymbol{N}_{i}
$$

The dilute approximation in the resistivity formulation is referred to as NIA, the non-interaction approximation,

$$
\boldsymbol{r}^{\mathrm{NIA}}=\boldsymbol{r}_{0}+\varphi_{i} \boldsymbol{H}_{i}
$$

where $\boldsymbol{H}_{i}$ denotes the resistivity contribution tensor (see appendix 6 for detailed expressions of $\boldsymbol{N}_{i}$ and the $\boldsymbol{N}_{i}$ tensors). The Mori-Tanaka-Benveniste 
$[21,22]$ estimate is written as (see also the recent formulation [23] and applications in elasticity)

$$
\begin{gathered}
\boldsymbol{\sigma}^{\mathrm{MTB}}=\boldsymbol{\sigma}_{0}+\left(\left(\boldsymbol{\sigma}_{i}-\boldsymbol{\sigma}_{0}\right)^{-1}+\frac{1-\varphi_{i}}{\varphi_{i}} \boldsymbol{N}_{i}^{-1}\right)^{-1} \\
\boldsymbol{r}^{\mathrm{MTB}}=\boldsymbol{r}_{0}+\left(\left(\boldsymbol{r}_{i}-\boldsymbol{r}_{0}\right)^{-1}+\frac{1-\varphi_{i}}{\varphi_{i}} \boldsymbol{H}_{i}^{-1}\right)^{-1}
\end{gathered}
$$

The Maxwell approximation, reformulated in terms of the property contribution tensors [24], leads to the formulas

$$
\begin{aligned}
& \boldsymbol{\sigma}^{\mathrm{MX}}=\boldsymbol{\sigma}_{0}+\varphi_{i}\left(\boldsymbol{N}_{i}^{-1}-\varphi_{i} \boldsymbol{P}_{\Omega}\right)^{-1} \\
& \boldsymbol{r}^{\mathrm{MX}}=\boldsymbol{r}_{0}+\varphi_{i}\left(\boldsymbol{H}_{i}^{-1}-\varphi_{i} \boldsymbol{Q}_{\Omega}\right)^{-1}
\end{aligned}
$$

where $\boldsymbol{P}_{\Omega}$ and $\boldsymbol{Q}_{\Omega}$ denote Hill tensors related to the effective ellipsoidal inclusion of the Maxwell scheme. See in [25] a discussion on the appropriate shape of the effective inclusion in the case of an isotropic host matrix. The Mori-Tanaka-Benveniste $M T B$ approximation corresponds to the particular case of Maxwell's approximation when $\boldsymbol{P}_{\Omega}=\boldsymbol{P}_{i}^{0}$. This is the case when the ellipsoidal distribution of one family of inclusions is aligned with and has the same shape as the inclusions. The generalization of the $M T B$ and Maxwell approximations to $N_{\text {inc }}$ families is written as [26]

$$
\boldsymbol{\sigma}^{\mathrm{MTB}}=\boldsymbol{\sigma}_{0}+\left(\sum_{i=1}^{N_{i n c}} \varphi_{i} \boldsymbol{N}_{i}\right) \cdot\left(\sum_{i=1}^{N_{i n c}} \varphi_{i}\left(\boldsymbol{\sigma}_{i}-\boldsymbol{\sigma}_{0}\right)^{-1} \cdot \boldsymbol{N}_{i}+\left(1-\sum_{i=1}^{N_{i n c}} \varphi_{i}\right) \boldsymbol{i}\right)^{-1}
$$

The Maxwell and Ponte Castañeda Willis [27] ( $P C W)$ approximations coincide (see [26]), the latter one being widely used in micromechanics of heterogeneous and cracked materials to account for spatial correlations between inclusions and/or cracks. The Hill tensors $\boldsymbol{P}_{\Omega}$ and $\boldsymbol{Q}_{\Omega}$ related to the shape of the effective inclusion of the Maxwell approximation correspond to the Hill tensor $\boldsymbol{P}_{d}$, representing the spatial correlation between inclusions, introduced in [27]. It must be emphasized that these tensors are defined within the assumption of ellipsoidal symmetry for the distribution of the inclusions. See in [28] a recent and detailed discussion on this issue, extensions 
of the $P C W$ approximation, and applications to linear and non-linear effective properties. In the present paper, only a sensitivity study on the shape of effective inclusions is presented by considering different aspect ratios in the particular case of spheroids. This may be considered as a first approach, without connection to the spatial distributions of inhomogeneities, to evaluate its importance. This key issue will be investigated in detail in further works.

$$
\boldsymbol{\sigma}^{\mathrm{MX}}=\boldsymbol{\sigma}_{0}+\left(\left(\sum_{i=1}^{N_{i n c}} \varphi_{i} \boldsymbol{N}_{i}\right)^{-1}-\boldsymbol{P}_{\Omega}\right)^{-1}
$$

\subsection{Differential Effective Method}

The differential scheme or differential effective method $D E M$, with application to elasticity and conductivity [29], is certainly the most widely used Effective Media Theory approximation in geophysics for the effective electrical-thermal conductivity and permittivity of geomaterials $[30,6]$. The differential equation for the conductivity tensor is written as

$$
\begin{gathered}
(1-x) \frac{d \boldsymbol{\sigma}}{d x}=\left(\boldsymbol{\sigma}_{i}-\boldsymbol{\sigma}\right) \cdot \boldsymbol{A}_{i} \\
\boldsymbol{A}_{i}=\left(\boldsymbol{i}+\boldsymbol{P}_{i} \cdot\left(\boldsymbol{\sigma}_{i}-\boldsymbol{\sigma}\right)\right)^{-1}, \quad \boldsymbol{P}_{i}=\boldsymbol{P}\left(\gamma_{i}, \boldsymbol{\sigma}\right)
\end{gathered}
$$

The case $x=0$ corresponds to the matrix phase

$$
\boldsymbol{\sigma}(x=0)=\boldsymbol{\sigma}_{0}
$$

It must be emphasized that in the general anisotropic case, a numerical integration of equation (11) is necessary, as the host matrix conductivity tensor $\boldsymbol{\sigma}$ varies along the integration path; in particular, the anisotropic ratio depends on the shape, orientation, and volume fraction of inclusions: $\boldsymbol{\sigma}=\boldsymbol{\sigma}(x)$ and $\boldsymbol{P}_{i}=\boldsymbol{P}_{i}\left(\gamma_{i}, \boldsymbol{\sigma}\right)$. The concentration tensor is then a function of the host matrix conductivity tensor $\boldsymbol{A}_{i}=\boldsymbol{A}_{i}(\boldsymbol{\sigma})$. The differential equation (11) has been solved by using the classical Runge-Kutta method (fourth-order Runge-Kutta method integration scheme, generally referred to as $R K_{4}$ ). 


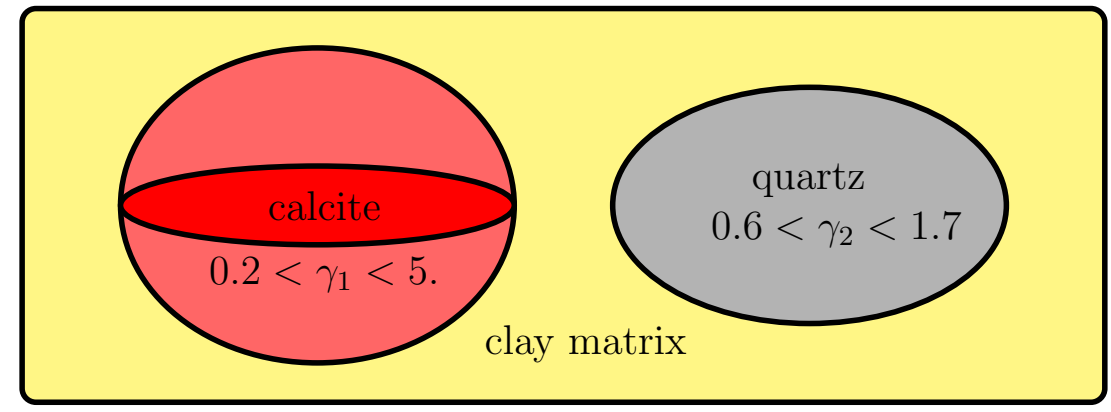

Figure 1: Reference three-phase model for Callovo-Oxfordian mudstone

\section{Reference microstructural data for a Callovo-Oxfordian mud- stone}

Consider a transversely isotropic clay rock, a Callovo-Oxfordian mudstone from the Paris sedimentary basin, which is extensively studied in France as a potential host rock for high-level radioactive waste repositories; see details in $[12,16,6]$. We will use the reference data set presented in [6], and the objective is to propose a new interpretation and inversion of experimental data based on a more accurate microstructural model, taking into account the anisotropy of the matrix and the shape and orientation of mineral inclusions in the clay matrix. The reference data used to characterize the microstructure of this rock at the mesoscale are extracted from the mineral map presented in figure 9 and references $[12,31]$. The clay mudstone is a multicomposite rock, but only the three main phases will be investigated for the sake of simplicity. At the mesoscale, one considers a three-phase domain composed of a clay matrix (index 0 ) with embedded solid mineral inclusions of calcite (index 1) and quartz (index 2) with respective volume fractions of $\varphi_{0}=0.60$, $\varphi_{1}=0.25$, and $\varphi_{2}=0.15$.

The conductive phase in the electrical conductivity problem is only the clay matrix (index 0). Quartz (index 2) and carbonate (index 1) inclusions are perfectly insulating phases with zero electrical conductivity. As aspect ratios $\gamma_{i}$ have been estimated on the basis of a $2 D$ mineral map, both flatted (oblate) and elongated (prolate) cases are considered for the $3 D$ approximation by spheroids. It is known in the isotropic case that for the considered range of aspect ratios $\gamma_{i}$, the sensitivity to shape is higher in the oblate case (flatted inclusions) than in the prolate case (elongated inclusions), 


\begin{tabular}{|c|c|c|c|}
\hline & clay matrix & calcite & quartz \\
\hline volume fraction $\varphi_{i}$ & $\varphi_{0}=0.60$ & $\varphi_{1}=0.25$ & $\varphi_{2}=0.15$ \\
\hline aspect ratio $\gamma_{i}$ (oblate case) & & $0.2<\gamma_{1}<1$. & $\gamma_{2}=0.6$ \\
aspect ratio $\gamma_{i}$ (prolate case) & & $1 .<\gamma_{1}<5$. & $\gamma_{2}=1.7$ \\
\hline
\end{tabular}

Table 1: Reference data set for Callovo-Oxfordian mudstone (from core sample EST205$709[4,6]$

all other parameters being equal. The reference macroscopic electrical conductivities are $\sigma_{T}^{\text {eff }}=9.310^{-1} \mathrm{~S} \mathrm{~m}^{-1}$ for the transverse (or horizontal) and $\sigma_{N}^{e f f}=2.810^{-1} \mathrm{~S} \mathrm{~m}^{-1}$ for the normal (or vertical) electrical conductivities, with the effective conductivity ratio $\sigma_{T}^{\text {eff }} / \sigma_{N}^{\text {eff }} \approx 3.3$. These values correspond to experimental measurements obtained from a two-electrode device (see details in [12]). The posed inverse problem is to determine the best fit of the two components of the electrical conductivities of the clay matrix phase $\sigma_{T}^{0}$ and $\sigma_{N}^{0}$ by taking into account microstructural information and the effective medium approximation. More precisely, as inclusion phases (calcite and quartz) are considered as perfect insulators in the electrical conductivity problem, the only parameter to fit with input data is the anisotropic ratio of the matrix conductivity tensor by adequately using proper microstructural information and the effective anisotropic ratio

$$
\frac{\sigma_{T}^{\mathrm{eff}}}{\sigma_{N}^{\mathrm{eff}}} \approx 3.3 \pm \varepsilon
$$

As the anisotropic ratio has been determined on a core sample (cm size, see $[12,6])$, the variability of natural mudstone properties implies that it is necessary to account for the uncertainty $\varepsilon$ on this parameter (this issue is addressed in section 4.2.2). It must be emphasized that the anisotropic ratio of electrical conductivity has been determined on the same core sample (EST05-709) as the one used for the mineral map.

\section{Numerical results and discussion}

We first present a sensitivity study of the microstructural model by increasing the degree of complexity: first, a two-phase model is considered 
(section 4.1), with only one inclusion phase, and second, a three-phase model is considered, with two different inclusion phases (calcite and quartz, section 4.2). In all cases, inclusion phases are considered to be perfect insulators (see [6]), but differences may be found in the shape and orientation distributions. The results for the anisotropic ratio of the transverse to normal conductivities of the matrix phase are presented.

The basic building block of the various homogenization techniques considered in this paper is the analytical solution for the electrical conductivity contribution tensor for an arbitrarily oriented isolated ellipsoidal inhomogeneity embedded in a transversely isotropic matrix. It uses as the reference the known reference solution from the Newtonian potential and a particular coordinate transform described in $[13,14,15]$. It only requires the solution of elliptic integrals and the algorithm for eigenvectors and eigenvalues of a symmetric $3-3$ matrix, for example, using Python-related numerical tools, Scientific Computing Tools for Python (scipy: scipy.special.ellipkinc and scipy.special.ellipeinc), and Numerical Python (numpy: linalg.eig). We present in the appendix 6 an unpublished derivation of the solution based on the Fourier transform method and a change of variable on the unit sphere similar to that presented for a similar elastic solution in [? ]. Details on the numerical integration of averaged tensors are presented in section 7. Averaged tensors and effective conductivity tensors are transversely isotropic with the same symmetry axis as the matrix.

4.1. Preliminary results with a simple two-phase matrix composite: one inclusion phase is considered

Simple limiting cases are considered for the two-phase model:

- aligned spheroidal inclusions with the same symmetry axis as the transversely isotropic matrix (case $a$ )

- spheroidal inclusions with the symmetry axis perpendicular to the symmetry axis of the matrix, randomly oriented in the transversely isotropic plane (case $b$ )

- randomly oriented spheroidal inclusions (case $c$ )

- an orientation distribution defined by an orientation distribution function $(O D F)$ that allows covering all intermediates cases between cases $a$ and $c$ (referred to as case $d$ ) 
The orientation distribution corresponding to case $b$ has been considered by [1] in a different context to represent microcracks induced in transversely isotropic rocks submitted to mechanical compression tests, verifying the symmetry of revolution about the symmetry axis of the material (the effective material is transversely isotropic, both in the initial and microcracked states). This case has been considered, as it is more relevant than case $a$ for elongated inhomogeneities of a prolate shape. In contrast, case $a$ is more useful than case $b$ for flatted inhomogeneities. Microstructural observations of mineral maps and scanning electron microscopy $S E M$ images show that elongated shapes are preferentially oriented along the transversely isotropic plane (see [5]). Case $a$ with the elongated spheroidal inhomogeneities corresponds to inhomogeneities perpendicular to the one observed. In case $b$, a random orientation distribution in the transverse plane matrix has been considered for two main reasons. The first is theoretical; it allows verifying the assumption of the transverse isotropy of the effective material, and one family of aligned spheroidal inhomogeneities with a symmetry axis perpendicular to the symmetry axis of the transversely isotropic matrix would induce an orthotropic effective material (see details in the appendix). The second is experimental: random or quasi-random orientation distributions of carbonates and quartz inhomogeneities in the transversely isotropic plane have been observed, at the mesoscale, for the reference Callovo-Oxfordian mudstone investigated in this paper (see [5]).

\subsubsection{One family of spheroidal insulating inhomogeneities: limiting cases of} aligned and random orientation distributions (cases $a, b$, and $c$ )

We first consider the simplest case $a$ of one family of perfectly insulating $\left(\boldsymbol{\sigma}_{i}=\mathbf{0}\right)$ spheroidal inhomogeneities aligned with the direction of symmetry of the transversely isotropic host matrix (same symmetry axis $\underline{n}$ for the inhomogeneities and matrix conductivity tensor). This case leads to analytical expressions for the effective conductivity tensor in the case of explicit schemes (MTB and Maxwell schemes will be detailed).

$$
\boldsymbol{\sigma}_{0}=\sigma_{0}\left(\nu^{2} \boldsymbol{i}_{T}+\boldsymbol{i}_{N}\right), \quad \boldsymbol{i}_{N}=\underline{n} \otimes \underline{n}, \quad, \quad \boldsymbol{i}_{T}=\boldsymbol{i}-\boldsymbol{i}_{N}
$$

where $\nu^{2}=\sigma_{T}^{0} / \sigma_{N}^{0}$ denotes the ratio of the transverse $\left(\sigma_{T}^{0}=\nu^{2} \sigma_{0}\right)$ to normal $\left(\sigma_{N}^{0}=\sigma_{0}\right)$ matrix conductivity. In sedimentary rocks and clay-based materials, one has $1 \leq \nu$ (see data in [32]). The shape of the effective inclusion in 


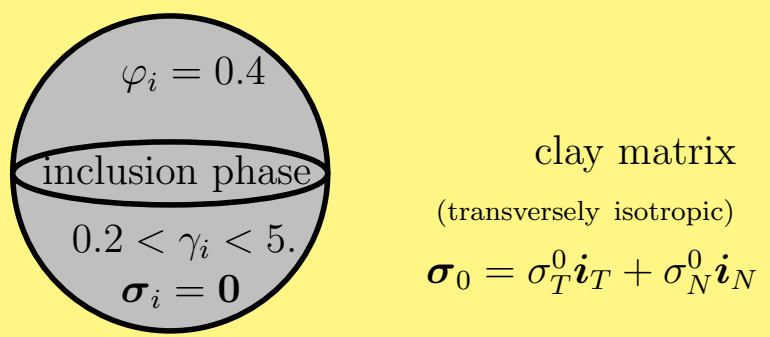

Figure 2: Simplified two-phase model with one family of aligned inclusions (case a).

the Maxwell approximation needs to be properly defined in the transversely isotropic case, as investigated in [26]. One respectively denotes the aspect ratios of the inhomogeneities and effective inclusion by $\gamma_{i}$ and $\gamma_{\Omega}$. The related Hill tensors are written as

$$
\boldsymbol{P}_{i}^{0}=\frac{1}{\sigma_{0}}\left(\frac{g_{i}}{\nu^{2}} \boldsymbol{i}_{T}+\left(1-2 g_{i}\right) \boldsymbol{i}_{N}\right), \quad \boldsymbol{P}_{0}^{\Omega}=\frac{1}{\sigma_{0}}\left(\frac{g_{\Omega}}{\nu^{2}} \boldsymbol{i}_{T}+\left(1-2 g_{\Omega}\right) \boldsymbol{i}_{N}\right)
$$

with coefficients $g_{i}=g\left(\nu \gamma_{i}\right)$ and $g_{\Omega}=g\left(\nu \gamma_{\Omega}\right)$ (function $g(t)$ is discussed in the appendix).

$$
\boldsymbol{\sigma}^{\mathrm{MX}}=\sigma_{T}^{\mathrm{MX}} \boldsymbol{i}_{T}+\sigma_{N}^{\mathrm{Mx}} \boldsymbol{i}_{N}
$$

The Maxwell approximation is written as

$$
\frac{\sigma_{T}^{\mathrm{MX}}}{\sigma_{0}}=\nu^{2} \frac{1-g_{i}-\varphi_{i}\left(1-g_{\Omega}\right)}{1-g_{i}+\varphi_{i} g_{\Omega}}, \quad \frac{\sigma_{N}^{\mathrm{MX}}}{\sigma_{0}}=\frac{2\left(g_{i}-\varphi_{i} g_{\Omega}\right)}{2 g_{i}+\varphi_{i}\left(1-2 g_{\Omega}\right)}
$$

In the particular case of the MTB approximation, $\gamma_{\Omega}=\gamma_{i}$ (and then, $g_{\Omega}=$ $\left.g_{i}\right)$; it becomes

$$
\frac{\sigma_{T}^{\mathrm{MTB}}}{\sigma_{0}}=\nu^{2} \frac{\left(1-g_{i}\right)\left(1-\varphi_{i}\right)}{1-\left(1-\varphi_{i}\right) g_{i}}, \quad \frac{\sigma_{N}^{\mathrm{MTB}}}{\sigma_{0}}=\frac{2 g_{i}\left(1-\varphi_{i}\right)}{2 g_{i}\left(1-\varphi_{i}\right)+\varphi_{i}}
$$

In the case of the isotropic host matrix, $\nu=1$, and the spherical shape, $\gamma_{\Omega}=\gamma_{i}=1$, one has $g_{i}=g_{\Omega}=1 / 3$, and the well-known formula for insulating spheres embedded in an isotropic matrix is recovered,

$$
\frac{\sigma_{T}^{\mathrm{MTB}}}{\sigma_{0}}=\frac{\sigma_{N}^{\mathrm{MTB}}}{\sigma_{0}}=\frac{2\left(1-\varphi_{i}\right)}{2+\varphi_{i}}
$$


In contrast to the $M T B$ approximation, the Maxwell model may induce zero effective conductivities for aligned spheroidal insulating inclusions, depending on the aspect ratios $\gamma_{i}$ and $\gamma_{\Omega}$, the volume fraction of the inhomogeneities $\varphi_{i}$ and the anisotropic degree of the matrix conductivity tensor through parameter $\nu$. The non-singularity conditions of Maxwell approximations of the transverse and normal conductivity are written as

$$
\begin{gathered}
\sigma_{T}^{\mathrm{MX}}>0, \quad 1-g\left(\nu \gamma_{\Omega}\right)<\frac{1-g\left(\nu \gamma_{i}\right)}{\varphi_{i}} \\
\sigma_{N}^{\mathrm{MX}}>0, \quad g\left(\nu \gamma_{\Omega}\right)<\frac{g\left(\nu \gamma_{i}\right)}{\varphi_{i}}
\end{gathered}
$$

In the case of transversely isotropic rocks, one generally has $1 \leq \nu$. For data representative of the clay rocks investigated in this paper, relation (22) for the normal conductivity needs to be accounted for; the singularity condition for transverse conductivity is never reached. A comparison between the model and experimental data leads to the following equation to be solved for the Maxwell approximation:

$$
\frac{\sigma_{N}^{\mathrm{MX}}}{\sigma_{T}^{\mathrm{MX}}}=\frac{\sigma_{N}^{\mathrm{eff}}}{\sigma_{T}^{\mathrm{eff}}}
$$

and then,

$$
\frac{1}{\nu^{2}} \frac{2\left(g_{i}-\varphi_{i} g_{\Omega}\right)}{2 g_{i}+\varphi_{i}\left(1-2 g_{\Omega}\right)} \frac{1-g_{i}+\varphi_{i} g_{\Omega}}{1-g_{i}-\varphi_{i}\left(1-g_{\Omega}\right)}=\frac{\sigma_{N}^{\mathrm{eff}}}{\sigma_{T}^{\mathrm{eff}}}
$$

The corresponding relation for the $M T B$ approximation is written as

$$
\frac{1}{\nu^{2}} \frac{2 g_{i}}{1-g_{i}} \frac{1-\left(1-\varphi_{i}\right) g_{i}}{2 g_{i}\left(1-\varphi_{i}\right)+\varphi_{i}}=\frac{\sigma_{N}^{\mathrm{eff}}}{\sigma_{T}^{\mathrm{eff}}}
$$

Similar relations have been derived for the differential scheme. scipy.optimize.brentq functions have been used to solve equations (24-25) and related equations for other schemes and orientation distributions. The ratios of the normal to transverse matrix conductivity predicted by the $M T B, D E M$ and Maxwell approximations are presented in figures 10-12. A comparison between the $M T B$ and Maxwell approximations shows that the influence of the spatial distribution of the inhomogeneities may be significant in the low range of the 
aspect ratio (flatted inhomogeneities). The shape of the effective inclusion of the Maxwell approximation needs to be carefully defined in the frame of a transversely isotropic host matrix. The aligned distribution of inclusions overestimates the influence of inclusions on the overall anisotropy and consequently underestimates that of the matrix for the lowest value of the aspect ratio $\gamma_{i}$. The expected lower bound of the matrix anisotropic ratio obtained for $\gamma_{i}=0.2$ is approximately $\nu^{2}=\sigma_{T}^{0} / \sigma_{N}^{0} \approx 2$ for the $M T B$ approximation. Cases $b$ and $c$ correspond to different averaging over orientation, respectively,

\begin{tabular}{|c|c|c|}
\hline & clay matrix & inhomogeneities \\
\hline volume fraction $\varphi_{i}$ & $\varphi_{0}=0.60$ & $\varphi_{i}=0.4$ \\
\hline aspect ratio $\gamma_{i}$ (oblate case) & & $0.2<\gamma_{i}<1$. \\
\hline aspect ratio $\gamma_{i}$ (prolate case) & & $1 .<\gamma_{i}<5$. \\
\hline
\end{tabular}

Table 2: Case 1: one family of aligned insulating inhomogeneities

transverse isotropization about the symmetry axis of the matrix (axially random case) and full isotropization (fully random case in $3 D$ ). The $M T B$ and Maxwell approximations in both cases are written as

$$
\begin{gathered}
\boldsymbol{\sigma}^{\mathrm{MTB}}=\boldsymbol{\sigma}_{0}+\left(\left(\boldsymbol{\sigma}_{i}-\boldsymbol{\sigma}_{0}\right)^{-1}+\frac{1-\varphi_{i}}{\varphi_{i}}\left(\overline{\boldsymbol{N}_{i}}\right)^{-1}\right)^{-1} \\
\boldsymbol{\sigma}^{\mathrm{MX}}=\boldsymbol{\sigma}_{0}+\varphi_{i}\left(\left(\overline{\boldsymbol{N}_{i}}\right)^{-1}-\varphi_{i} \boldsymbol{P}_{\Omega}\right)^{-1}
\end{gathered}
$$

$\overline{\boldsymbol{N}_{i}}$ denotes the conductivity contribution tensor averaged over orientation. As indicated, $\overline{\boldsymbol{N}_{i}}$ is different between the two cases $b$ and $c$, and respective calculations are detailed in the appendix 7 . The results for case $b$ with the $D E M$ and $M T B$ approximations are presented in figure 11. For materials such as the transversely isotropic reference mudstone studied in this paper, this case can be considered a relevant continuation of the aligned case $a$ in the prolate range. The known result in the isotropic case still holds under transverse isotropy: the effect of the shape on the effective conductivity is higher in the oblate range (flatted inhomogeneities) than in the prolate range 
(elongated inhomogeneities). In case $c$, with one family of randomly oriented insulating inhomogeneities of spheroidal shape embedded in a transversely isotropic matrix, the only source of overall anisotropy is the matrix. An upper bound of the anisotropic ratio of the matrix phase can be estimated by considering the lowest value of the aspect ratio of the inhomogeneities $\left(\gamma_{i}=0.2\right)$. We do not consider for this estimate case $b$ with an axially random distribution, as it corresponds to an unrealistic orientation distribution in the low range of the aspect ratio of the oblate case (as previously mentioned, the distribution $b$ is more useful in the prolate case). The Hill tensor of an arbitrarily oriented ellipsoidal inhomogeneity embedded in a transversely isotropic matrix is calculated by using an exact solution [13]. It yields equations (with $(i)=(b),(c)$ )

$$
\frac{\sigma_{N}^{\mathrm{MTB}(i)}}{\sigma_{T}^{\mathrm{MTB}(i)}}=\frac{\sigma_{N}^{\mathrm{eff}}}{\sigma_{T}^{\mathrm{eff}}} \quad, \quad \frac{\sigma_{N}^{\mathrm{MX}(i)}}{\sigma_{T}^{\mathrm{MX}(i)}}=\frac{\sigma_{N}^{\mathrm{eff}}}{\sigma_{T}^{\mathrm{eff}}}
$$

which needs to be inverted to obtain the matrix conductivity ratio in each case. The results are presented in figures 13-14. The expected upper bound of the matrix anisotropic ratio obtained for $\gamma_{i}=0.2$ is approximately $\nu^{2}=$ $\sigma_{T}^{0} / \sigma_{N}^{0} \approx 4.7$ for the $M T B$ approximation.

$$
2.0 \leq\left(\frac{\sigma_{T}^{0}}{\sigma_{N}^{0}}\right)^{\mathrm{MTB}} \leq 4.7
$$

The same comment as in the aligned case can be made, and differences between the $M T B$ and Maxwell approximations are significant in the low range of the aspect ratio. This result underlines the potential influence of the spatial distribution of inclusions in the case of elongated inclusions. The differential scheme yields the following bounds:

$$
1.3 \leq\left(\frac{\sigma_{T}^{0}}{\sigma_{N}^{0}}\right)^{\mathrm{DEM}} \leq 6.0
$$

In the low aspect ratio range of inclusions, the $M T B$ approximation underestimates interactions between inclusions and their effect on the overall conductivity compared to the DEM and Maxwell approximations. In the case of the Maxwell approximation, the shape of the effective inclusion needs to be carefully defined for inclusions with an aspect ratio in the low range (see the relation 22), and the overall anisotropy strongly depends on the shape of 
the effective inclusion and the orientation and shape of inclusions. It can be observed in figure 14 that the axially random orientation distribution (case b) strongly overestimates the anisotropic ratio of the matrix conductivity for the lowest aspect ratio $\gamma_{i}=0.2$. This was not taken into account in previous evaluations of bounds because it does not correspond to the observed microstructure of such rocks for the lowest range of aspect ratios of carbonate inhomogeneities.

\subsubsection{One family of spheroidal insulating inhomogeneities with orientation defined by $O D F$}

This is the intermediate case, between cases $a$ and $c$, and the most realistic in comparison to available microstructural data. Most importantly, it can allow an admissible range for the orientation distribution to be defined and a more accurate account of the effect of the orientation of inclusions on the overall anisotropy of the electrical conductivity tensor. Relations (26-27) apply, with the only difference being the calculation of averaged tensors $\overline{\boldsymbol{A}_{i}}$ and $\overline{\boldsymbol{N}_{i}}$.

$$
\begin{gathered}
\overline{\boldsymbol{B}}=\bar{B}_{k l} \underline{e}_{k} \otimes \underline{e}_{l} \\
\bar{B}_{k l}=\frac{1}{4 \pi} \int_{\varphi_{\alpha}=0}^{2 \pi} \int_{\theta_{\alpha}=0}^{\pi} B_{k l}\left(\varphi_{\alpha}, \theta_{\alpha}\right) W\left(\theta_{\alpha}, \varphi_{\alpha}\right) \sin \theta_{\alpha} d \theta_{\alpha} d \varphi_{\alpha}
\end{gathered}
$$

where $W\left(\theta_{\alpha}, \varphi_{\alpha}\right)$ denotes the orientation distribution function, (ODF)

$$
\frac{1}{2 \pi} \int_{\theta_{\alpha}=0}^{\pi} \int_{\varphi_{\alpha}=0}^{2 \pi} W\left(\theta_{\alpha}, \varphi_{\alpha}\right) \sin \left(\theta_{\alpha}\right) d \theta_{\alpha} d \varphi_{\alpha}=1
$$

In this paper, we focus on the transversely isotropic case and then on the $O D F$ case, respecting the symmetry of revolution (same symmetry axis as the matrix). $O D F$ only depends on the spherical angle $\theta_{\alpha}$ (colatitude angle relative to symmetry axis $\underline{n}=\underline{e}_{3}$ ) and parameters defining the degree of preferential alignment $W=W\left(\theta_{\alpha}\right)$.

$$
\int_{0}^{\pi / 2} W\left(\theta_{\alpha}\right) \sin \left(\theta_{\alpha}\right) d \theta_{\alpha}=1
$$

One considers a $\pi$ periodic Gaussian-like function [16]

$$
W\left(\theta_{\alpha}, \chi\right)=\frac{\chi \cosh \left(\chi \cos \left(\theta_{\alpha}\right)\right)}{\sinh (\chi)}
$$


where $\chi$ is the degree of preferential alignment, and $\chi=0$ and $\chi=\infty$, respectively, correspond to random and aligned orientation distributions; see figure 15. See also in [23] a review of $O D F$ and an example of reconstruction of the orientation distribution function on the basis of computed tomography in the case of a short-fibre-reinforced composite. The results for the inverted anisotropic ratio of the matrix phase are presented in figure 16 for the case of the $M T B$ and DEM approximations. The respective influences of the shape of inclusions and their orientation distribution on the anisotropic ratio of the matrix can be evaluated. The $O D F$ case is more realistic and allows the entire range to be covered, including aligned and random distributions. As expected, inversion of the matrix anisotropic ratio does not depend on the shape and orientation of inclusions in the upper range of the inclusion aspect ratio $0.8<\gamma_{i}<1$ in the oblate case, a nearly spherical shape. The sensitivity of the matrix anisotropic ratio to the aspect ratio is lower in the prolate case (elongated inclusions) compared to the previous oblate case, and the same observation still holds in the range of $1<\gamma<2$.

4.2. Results for the three-phase matrix composite: calcite and quartz inclusion phases are considered

Subsection 4.2.1 presents results for the reference three-phase model, considering the four cases of orientation distributions: the limiting cases of aligned and random, completed by the intermediate case of the preferred alignment defined by $O D F$. As the Callovo-Oxfordian mudstone is a natural rock with important variability of properties, the variability with depth and the lateral variability are assessed by comparing different boreholes; see [31]. We complete the numerical results with two sensitivity studies on

- the anisotropic ratio of the macroscopic electrical conductivity tensor (subsection 4.2.2); this study is related to the previously discussed uncertainty of this macroscopic parameter.

- the mineralogical composition (subsection 4.2.3); the three-phase reference model previously considered on the basis of [6] is completed by two reference models, extracted from [31], including a highly carbonated case. 


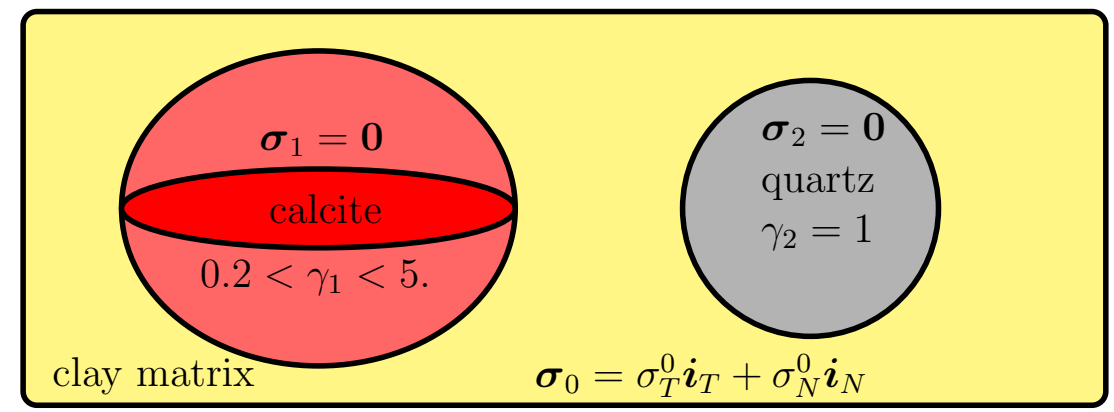

Figure 3: Reference three-phase model with two families of inclusions

\subsubsection{Two families of spheroidal insulating inhomogeneities with orientation defined by $O D F$}

This section considers the reference three-phase model defined in table (3), composed of a transversely isotropic clay matrix and two distinct families of insulating inclusions (calcite and quartz). Since the contribution to the effective conductivity of oblate spheroidal inclusions with aspect ratio $\gamma_{2}=$ 0.6 or prolate inclusions with aspect ratio $\gamma_{2}=1.7$ and a random orientation distribution is very close to that of spherical inclusions, the quartz family is simplified, and quartz inclusions are taken as spherical.

The analysis is then focused on the calcite and clay matrix phases, and the main issue is to evaluate their respective contributions to the overall anisotropy. We use relations (36) and (10), with

$$
\sum_{i=1}^{N_{\text {inc }}} \varphi_{i} \boldsymbol{N}_{i}=\varphi_{1} \overline{\boldsymbol{N}_{1}}+\varphi_{2} \boldsymbol{N}_{2}, \quad 1-\sum_{i=1}^{N_{\text {inc }}} \varphi_{i}=1-\varphi_{1}-\varphi_{2}
$$

and $O D F$ is taken into account to calculate average tensors of the calcite phase $\overline{\boldsymbol{N}_{c}}$. In the case of two families of inhomogeneities, the construction path of the differential scheme needs to be discussed, as effective properties depend on it (see [33]). For the sake of simplicity, as quartz and carbonates inclusions are roughly of the same scale and of similar size, the simplest homothetic path has been considered for DEM.

The three-phase model leads to new bounds on the anisotropic ratio of the matrix phase; as expected, these bounds are narrower compared to previous 


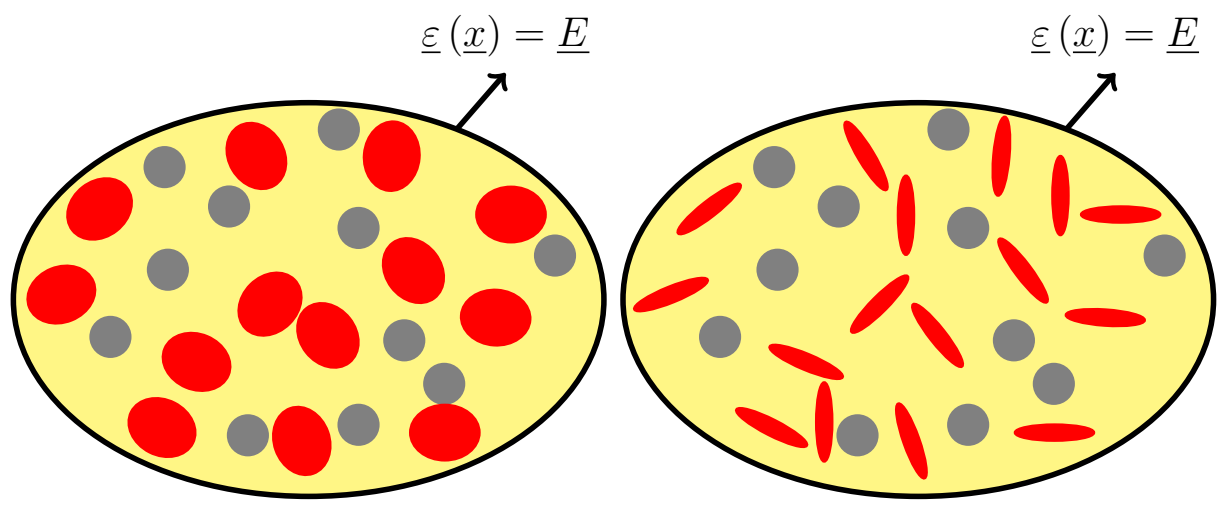

Figure 4: $2 D$ sketch of the $3 D$ microstructure. Three-phase model for Callovo-Oxfordian mudstone: transversely isotropic matrix with spherical inclusions of quartz and oriented oblate spheroidal inclusions of calcite (left $\gamma_{1}=0.8$, right $\gamma_{1}=0.2$, same volume fractions in both cases)

\begin{tabular}{|c|c|c|c|}
\hline & clay matrix & calcite & quartz \\
\hline volume fraction $\varphi_{i}$ & $\varphi_{0}=0.60$ & $\varphi_{1}=0.25$ & $\varphi_{2}=0.15$ \\
\hline aspect ratio (oblate case) $\gamma_{i}$ & & $0.2<\gamma_{1}<1$. & $\gamma_{2}=1$. \\
aspect ratio (prolate case) $\gamma_{i}$ & & $1 .<\gamma_{1}<5$. & $\gamma_{2}=1$. \\
\hline
\end{tabular}

Table 3: Reference model with two families of insulating inclusions. Application to electrical conductivity of Callovo-Oxfordian mudstone [6]

bounds (29-30) obtained for the simplified two-phase model

$$
2.5 \leq\left(\frac{\sigma_{T}^{0}}{\sigma_{N}^{0}}\right)^{\mathrm{MTB}} \leq 4.4 \quad, \quad 2.1 \leq\left(\frac{\sigma_{T}^{0}}{\sigma_{N}^{0}}\right)^{\mathrm{DEM}} \leq 5.2
$$

and the observations on the differences between the $M T B$ and DEM approximations still hold. The complete results for the anisotropic ratio are presented in figures 17-18. 


\subsubsection{Sensitivity study on the anisotropic ratio of the effective conductivity tensor}

A sensitivity study on the anisotropic ratio of the effective conductivity tensor $\sigma_{T}^{\text {eff }} / \sigma_{N}^{\text {eff }}$ is presented in this section to take into account uncertainty in this effective parameter due to the variability of the considered CallovoOxfordian mudstone. The range of the anisotropic ratio is $\nu_{\text {eff }}^{2}=\sigma_{T}^{\text {eff }} / \sigma_{N}^{\text {eff }}=$ $2.3-6.3$, with the reference data set value $\sigma_{T}^{\text {eff }} / \sigma_{N}^{\text {eff }} \approx 3.3$. The three-phase model has been considered, and the inverted anisotropic ratio of the matrix phase $\sigma_{T}^{0} / \sigma_{N}^{0}$ as a function of the effective ratio $\nu_{\text {eff }}^{2}$ for aspect ratios of the calcite inclusion phase $\gamma_{1}=0.2,0.3,0.4,1$ and in the limiting cases of random and aligned distributions for the $M T B$ and $D E M$ schemes is presented in figure 20. The influence of the aspect ratio $\gamma_{1}$ of calcite inclusions on the matrix anisotropy for a given ratio of the effective electrical conductivity is significant in the low range of the aspect ratio (by comparing $\gamma_{1}=0.2$ and 0.3$)$. The effect on the anisotropy of the random orientation distribution of spheroidal inclusions with aspect ratio $\gamma_{1}=0.4$ is very close to that of spherical inclusions. This well-known result in the isotropic case still holds in the transversely isotropic case. At a fixed inclusion aspect ratio, the anisotropic ratio of the matrix electrical conductivity tensor increases quasi-linearly with the overall anisotropic ratio. The complete results of the 3 -phase model with the degree of alignment of inclusions defined by $O D F$ and the effective anisotropic ratios $\nu_{\text {eff }}^{2}=\left(\nu_{\text {eff }}\right)^{2}=2.3,4.3$ are presented in figure 21. This figure underlines the combined significant influence of the orientation distribution of inclusions on the overall anisotropy in the low range of the aspect ratio $\gamma_{1}$, as previously discussed in the reference case $\nu_{\text {eff }}^{2}=\sigma_{T}^{\text {eff }} / \sigma_{N}^{\text {eff }}=3.3$.

These modelling findings suggest that for the investigated reference mudstone, the main source of the overall anisotropy of the electrical conductivity is found in the clay matrix and the details of its microstructure. The effect of the orientation distribution and shape of carbonate inhomogeneities on the overall anisotropy may be significant only in the low range of the aspect ratio.

\subsubsection{Sensitivity study on volume fractions of constituents}

The Callovo-Oxfordian mudstone (or argillite) studied in this paper is a natural rock with important variability in the mineral composition, vertically along the thickness $(130 . \mathrm{m})$ (see $[4,6,31])$ and horizontally if different boreholes are considered. On the basis of the latter reference, we define two 
reference models; model 1 corresponds to a high content of carbonates, and model 2 corresponds to average values deduced from X-ray diffractometry and calcimetry measurements; see table 4 . It must be emphasized that the effective electrical conductivities taken into account in this paper have been measured on a sample with a high volume fraction of clay $[12,6]$ from a drill core named EST05-709 (depth of $468.9 \mathrm{~m}$ ) retrieved from the Andra EST205 borehole. Model 1 corresponds to a highly carbonated layer, retrieved from the Andra EST20 borehole at a 494. m depth, and model 2 is closer to the reference model previously adopted in this paper and corresponds to $482.2 \mathrm{~m}$. Figure 22 presents the evolution of the matrix anisotropic ratio as a function of the effective anisotropic ratio $2.3<\nu_{\text {eff }}^{2}=\sigma_{T}^{\text {eff }} / \sigma_{N}^{\text {eff }}<6.3$, similar to the one presented in figure 20 for the reference model. It can be observed that the $M T B$ approximation underestimates the influence of the calcite inclusion phase on the overall anisotropy compared to DEM, with significant differences in the case of the high volume fraction of calcite (model 1). The results presented in 22 underline the high sensitivity of the anisotropy of the electrical conductivity tensor to the mineral composition, as expected. In particular, in the case of highly carbonated material (model 1), the main source of the overall anisotropy may be the carbonate phase in the low aspect ratio range, depending on the orientation distribution. This can be illustrated by the ratios

$$
\frac{\left(\frac{\sigma_{T}^{0}}{\sigma_{N}^{0}}\right)_{\text {random }}^{\mathrm{MTB}}}{\left(\frac{\sigma_{T}^{0}}{\sigma_{N}^{0}}\right)_{\text {aligned }}^{\mathrm{MTB}}} \frac{\left(\frac{\sigma_{T}^{0}}{\sigma_{N}^{0}}\right)_{\text {random }}^{\mathrm{DEM}}}{\left(\frac{\sigma_{T}^{0}}{\sigma_{N}^{0}}\right)_{\text {aligned }}^{\mathrm{DEM}}}
$$

which allow quantification of the potential effect of the carbonate phase (shape and orientation distribution) on the overall anisotropy. These ratios are equal to 1 in the case of spherical inhomogeneities: the overall anisotropy is only due to the clay matrix phase. From figure 22, it can be observed that the $D E M$ approximation predicts very high values (115., 7.0 and 3.1 for $\gamma_{1}=0.2,0.3$, and 0.4 , respectively), whereas the corresponding values for $M T B$ scheme remain limited to 3.4, 2.1 and 1.6. In this case, due to the high volume fraction of the carbonate phase, the differences between the DEM and $M T B$ results are significant, with the latter underestimating the effect of the inclusion phase on the overall anisotropy.The results for model 2 are quite similar to those presented for the reference model. 


\begin{tabular}{|c|c|c|c|}
\hline & clay matrix & calcite & quartz \\
\hline & $\varphi_{0}$ & $\varphi_{1}$ & $\varphi_{2}$ \\
model 1 & 0.34 & 0.53 & 0.13 \\
model 2 & 0.49 & 0.28 & 0.23 \\
reference model & 0.60 & 0.25 & 0.15 \\
\hline aspect ratio $\gamma_{i}$ & & $0.2<\gamma_{1}<0.8$ & $\gamma_{2}=1$ \\
\hline
\end{tabular}

Table 4: Mineral composition of 3 reference three-phase models, representative of different facies of the Callovo-Oxfordian mudstone [31]

\section{Conclusions}

The main novelty of the presented analysis and results in the context of anisotropic materials, such as the investigated transversely isotropic rocks, is as follows:

- an account is given of the arbitrarily oriented non-clay spheroidal inhomogeneities embedded in a transversely isotropic matrix, owing to the analytical solution [13].

- the separate influences of the matrix anisotropy and inhomogeneities distribution (shape and orientation) on the overall electrical conductivity are presented.

Simple limiting cases are considered, the aligned and random distributions, as well as a two-phase model with one inclusion phase family, which is useful as a first approach. This approach allows bounds on the unknown parameter, the anisotropic ratio of the transverse and normal electrical conductivities of the matrix, to be obtained. The effects of the orientation and shape of inclusions on the overall anisotropy are quantified. For the investigated Callovo-Oxfordian mudstone, the main source of the overall anisotropy of the electrical conductivity should be found in the matrix phase (orientation, shape of clay minerals, pores, etc.). The effect of non-clay inhomogeneities on the overall anisotropy may be significant in the low range of the aspect ratios of calcite inclusions, which corresponds to flatted shapes that are highly 
different from spherical shapes. The use of $O D F$ is promising in the sense that it enables

- microstructural data to be more adequately accounted for, for example, X-ray nanocomputed tomography, scanning electron microscopy $(S E M)$, and mineral map data, in the case of rock materials similar to the investigated mudstone.

- ranges of the orientation distributions of inclusion to be defined and then constraints on the inversion of the matrix property to be added.

The comparison between the reference approximation schemes MTB, Maxwell, and $D E M$ shows that similar to elasticity, $M T B$ underestimates interactions between inclusions and their effect on the overall conductivity compared to $D E M$ and Maxwell in the low range of the aspect ratio (approximately $\left.0.2<\gamma_{i}<0.5\right)$. The sensitivity of the inversion of the matrix property to the shape and orientation of inclusions underlines the need for microstructural investigations and the mapping techniques previously mentioned.

Ongoing work is focused on accounting for the spatial interactions between inhomogeneities and their effect on the effective electrical conductivity. A comparison between the single inclusion approach adopted in this paper and multipole expansion of the perturbation fields of inhomogeneities in terms of the ellipsoidal harmonics approach $[34,35]$ will be presented in a future paper. The latter approach allows explicit consideration of the spatial interactions between inhomogeneities and the corresponding semi-analytical solution [35] for the anisotropic case, which will be useful to validate the single-inclusion approach for the posed problem and investigated materials.

\section{Appendix. Polarization and concentration conductivity tensors related to a single ellipsoidal inclusion in an anisotropic matrix}

\subsection{Concentration tensors and property contribution tensors related to an ellipsoidal inclusion}

The reference dilute approximation in a conductivity formulation is useful in the low limit of the volume fraction of ellipsoidal inclusions $\varphi_{i} \ll 1$, written as

$$
\boldsymbol{\sigma}^{\mathrm{DIL}}=\boldsymbol{\sigma}_{0}+\varphi_{i}\left(\boldsymbol{\sigma}_{i}-\boldsymbol{\sigma}_{0}\right) \cdot \boldsymbol{A}_{i}
$$


with the dilute conductivity concentration tensor

$$
\boldsymbol{A}_{i}=\left(\boldsymbol{i}+\boldsymbol{P}_{i}^{0} \cdot\left(\boldsymbol{\sigma}_{i}-\boldsymbol{\sigma}_{0}\right)\right)^{-1}
$$

$\boldsymbol{P}_{i}^{0}$ denotes the Hill tensor of a single ellipsoidal inhomogeneity $i$ embedded in a infinite homogeneous matrix with conductivity tensor $\boldsymbol{\sigma}_{0}$ (see section 6). Relation (39) may be rewritten by using the conductivity contribution tensor $\boldsymbol{N}_{i}$

$$
\boldsymbol{\sigma}^{\mathrm{DIL}}=\boldsymbol{\sigma}_{0}+\varphi_{i} \boldsymbol{N}_{i}
$$

The conductivity $\boldsymbol{N}_{i}$ and resistivity $\boldsymbol{H}_{i}$ contribution tensors related to an isolated ellipsoidal inclusion embedded in an infinite matrix with conductivity tensor $\boldsymbol{\sigma}_{0}=\boldsymbol{r}_{0}^{-1}$ can be written as

$$
\boldsymbol{N}_{i}=\left(\boldsymbol{P}+\left(\boldsymbol{\sigma}_{i}-\boldsymbol{\sigma}_{0}\right)^{-1}\right)^{-1}, \quad \boldsymbol{H}_{i}=\left(\boldsymbol{Q}+\left(\boldsymbol{r}_{i}-\boldsymbol{r}_{0}\right)^{-1}\right)^{-1}
$$

where $\boldsymbol{Q}$ is related to the Hill polarization tensor $\boldsymbol{P}$ used in the conductivity formulation,

$$
\boldsymbol{Q}=\boldsymbol{\sigma}_{0} .\left(\boldsymbol{i}-\boldsymbol{P} . \boldsymbol{\sigma}_{0}\right)
$$

One has

$$
\boldsymbol{H}_{i}=-\boldsymbol{\sigma}_{0}^{-1} \cdot \boldsymbol{N}_{i} \cdot \boldsymbol{\sigma}_{0}^{-1}
$$

and

$$
\boldsymbol{N}_{i}=\left(\boldsymbol{\sigma}_{i}-\boldsymbol{\sigma}_{0}\right) \cdot \boldsymbol{A}_{i}
$$

The dilute conductivity concentration tensor can be written as

$$
\boldsymbol{A}_{i}=\left(\boldsymbol{i}+\boldsymbol{P} .\left(\boldsymbol{\sigma}_{i}-\boldsymbol{\sigma}_{0}\right)\right)^{-1}
$$

\subsection{Hill polarization tensor}

The shape tensor related to an ellipsoid $\varepsilon_{\mathcal{H}}$ of semi-axis length $a_{i}$ and unit vector $\underline{e}_{i}$ directed along the axis is written as

$$
\mathcal{H}=\sum_{i=1}^{3} a_{i} \underline{e}_{i} \otimes \underline{e}_{i}
$$

and the ellipsoid is described by the equation

$$
\underline{x} .\left({ }^{t} \mathcal{H} . \mathcal{H}\right)^{-1} \cdot \underline{x} \leq 1
$$

which is written as follows in the coordinate system attached to the ellipsoid:

$$
\underline{x} \in \varepsilon_{\mathcal{H}} \Leftrightarrow \frac{x_{1}^{2}}{a_{1}^{2}}+\frac{x_{2}^{2}}{a_{2}^{2}}+\frac{x_{3}^{2}}{a_{3}^{2}} \leq 1
$$


6.3. Solution for a single ellipsoidal inclusion arbitrarily oriented in an orthotropic matrix

We present in this section an alternative derivation based on the Fourier transform (unpublished presentation [36]) of the general solution [13] for the isolated ellipsoidal inclusion of an arbitrary orientation embedded in an orthotropic matrix. This approach uses techniques similar to those presented in reference book [37], in particular, a change of variable is useful to calculate the surface integral on the unit sphere in the frame of the Fourier transform method. The general solution for the polarization tensor of an ellipsoid arbitrarily oriented in an orthotropic matrix has been given in $[13,14]$ (see also [15] for a detailed analysis and application to fractured rocks).

$$
P_{i j}(\boldsymbol{\sigma}, \mathcal{H})=\frac{\partial^{2}}{\partial x_{i} \partial x_{j}} \int_{\varepsilon_{\mathcal{H}}} G\left(\underline{x}-\underline{x}^{\prime}\right) d \Omega_{x^{\prime}}
$$

$\boldsymbol{\sigma}$ : anisotropic conductivity of an infinite medium; $G$ : related Green function,

$$
G(\underline{x})=-\frac{1}{4 \pi \sqrt{\operatorname{det} \boldsymbol{\sigma}}} \frac{1}{\sqrt{\underline{x} \cdot \boldsymbol{\sigma}^{-1} \cdot \underline{x}}}
$$

In the case of and isotropic matrix, $\boldsymbol{\sigma}=\sigma \boldsymbol{i}$,

$$
G(\underline{x})=-\frac{1}{4 \pi \sigma} \frac{1}{\sqrt{\underline{x} \cdot \underline{x}}}=-\frac{1}{4 \pi \sigma} \frac{1}{\|\underline{x}\|}
$$

The reference solution from the Newtonian potential may be expressed as

$$
P_{i j}(\boldsymbol{i}, \boldsymbol{H})=-\frac{1}{4 \pi} \frac{\partial^{2}}{\partial x_{i} \partial x_{j}} \int_{\varepsilon_{\mathcal{H}}} \frac{1}{\left\|\underline{x}-\underline{x^{\prime}}\right\|} d \Omega_{x^{\prime}}, \quad \underline{x} \in \varepsilon_{\mathcal{H}}
$$

with semi-lengths (3D case: 1 major radius $a_{1}, 2$ aspect ratios $\eta$ and $\omega$ ) defined as follows:

$$
a_{1} \geq a_{2} \geq a_{3}>0 \quad, \quad 1 \geq \eta=a_{2} / a_{1} \geq \omega=a_{3} / a_{1}>0
$$

The shape tensor $\mathcal{H}$ defining ellipsoid $\varepsilon_{\mathcal{H}}$ is rewritten as

$$
\mathcal{H}=a_{1}\left(\underline{e}_{1} \otimes \underline{e}_{1}+\eta \underline{e}_{2} \otimes \underline{e}_{2}+\omega \underline{e}_{3} \otimes \underline{e}_{3}\right)
$$

The reference solution from the Newtonian potential in the frame attached to the ellipsoid is written as

$$
\boldsymbol{P}(\boldsymbol{i}, \boldsymbol{\mathcal { H }})=I_{1} \underline{e}_{1} \otimes \underline{e}_{1}+I_{2} \underline{e}_{2} \otimes \underline{e}_{2}+I_{3} \underline{e}_{3} \otimes \underline{e}_{3}
$$


with $I_{1}$ and $I_{3}$ given by incomplete elliptic integrals of the first and second kind.

$$
\begin{gathered}
I_{1}=\frac{\eta \omega}{\left(1-\eta^{2}\right) \sqrt{1-\omega^{2}}}(\mathcal{F}-\mathcal{E}) \\
I_{3}=\frac{\eta \omega}{\left(\eta^{2}-\omega^{2}\right) \sqrt{1-\omega^{2}}}\left(\frac{\eta \sqrt{1-\omega^{2}}}{\omega}-\mathcal{E}\right) \\
I_{2}=1-I_{1}-I_{3}
\end{gathered}
$$

$\mathcal{F}$ and $\mathcal{E}$ denote incomplete elliptic integrals of the first and second kind,

$$
\mathcal{F}(\theta, \kappa)=\int_{0}^{\theta} \frac{d t}{\sqrt{1-(\kappa \sin (t))^{2}}}, \quad \mathcal{E}(\theta, \kappa)=\int_{0}^{\theta} \sqrt{1-(\kappa \sin (t))^{2}} d t
$$

with amplitude $\theta$ and parameter $\kappa$ defined as

$$
\theta=\arcsin \left(\sqrt{1-\omega^{2}}\right), \quad \kappa=\sqrt{\frac{1-\eta^{2}}{1-\omega^{2}}}
$$

The particular cases of spheroids and a sphere, respectively, correspond to two distinct semi-lengths and one semi-length:

- prolate spheroid

$$
\begin{gathered}
a_{1}>a_{2}=a_{3}, \quad \text { aspect ratio } \quad \xi=\frac{a_{1}}{a_{3}}>1 \\
I_{2}=I_{3}=g(\xi), \quad I_{1}=1-2 g(\xi)
\end{gathered}
$$

- oblate spheroid

$$
\begin{gathered}
a_{1}=a_{2}>a_{3}, \quad \text { aspect ratio } \quad \xi=\frac{a_{3}}{a_{1}}<1 \\
I_{1}=I_{2}=g(\xi), \quad I_{3}=1-2 g(\xi)
\end{gathered}
$$


- sphere

$$
\begin{gathered}
a_{1}=a_{2}=a_{3}, \quad \text { aspect ratio } \xi=1 \\
I_{1}=I_{2}=I_{3}=g(1)=\frac{1}{3}
\end{gathered}
$$

with

$$
g(\xi)=\left\{\begin{array}{llr}
\frac{1}{2}\left(1+\frac{1}{u}\left(1-\frac{\xi}{\sqrt{v}} \arctan \left(\frac{\sqrt{v}}{\xi}\right)\right)\right) & \text { if } \quad \xi<1 \\
\frac{1}{3} & \text { if } \quad \xi=1 \\
\frac{1}{2}\left(1+\frac{1}{u}\left(1-\frac{\xi}{2 \sqrt{u}} \ln \left(\frac{\xi+\sqrt{u}}{\xi-\sqrt{u}}\right)\right)\right) & \text { if } \quad \xi>1 \\
v=1-\xi^{2}, \quad u=\xi^{2}-1 &
\end{array}\right.
$$

The Fourier transform method leads to a surface integral on the unit sphere,

$$
P_{i j}(\boldsymbol{\sigma}, \mathcal{H})=\frac{a_{1} a_{2} a_{3}}{4 \pi} \int_{S_{\eta}} \frac{\eta_{i} \eta_{j}}{\left(\sigma_{k l} \eta_{k} \eta_{l}\right)\left(a_{1}^{2} \eta_{1}^{2}+a_{2}^{2} \eta_{2}^{2}+a_{3}^{2} \eta_{3}^{2}\right)^{3 / 2}} d S_{\eta}
$$

with spherical coordinates $\eta_{i}\left(\eta_{1}^{2}+\eta_{2}^{2}+\eta_{3}^{2}=1\right)$ as follows:

$$
\begin{aligned}
& \eta_{1}=\cos \varphi \sin \theta, \quad \eta_{2}=\sin \varphi \sin \theta, \quad \eta_{3}=\cos \theta \\
& d S_{\eta}=\sin \theta d \theta d \varphi, \quad 0 \leq \theta \leq \pi, \quad 0 \leq \varphi \leq 2 \pi
\end{aligned}
$$

The derivation of the solution may be summarized as follows:

1 Introduce new coordinates on the unit sphere, replacing $\eta_{i}$ by $\zeta_{i}$ such that (see the change of variable used by [37] chap. 4, Anisotropic inclusions, relations 16.10 and 17.9 for the elastic problem)

$$
\begin{gathered}
\zeta_{i}=\frac{a_{i} \eta_{i}}{\zeta}, \quad \zeta=\left(a_{1}^{2} \eta_{1}^{2}+a_{2}^{2} \eta_{2}^{2}+a_{3}^{2} \eta_{3}^{2}\right)^{1 / 2} \\
\zeta_{1}^{2}+\zeta_{2}^{2}+\zeta_{3}^{2}=1, \quad d S_{\zeta}=\frac{a_{1} a_{2} a_{3}}{\zeta^{3}} d S_{\eta}
\end{gathered}
$$


This yields

$$
\boldsymbol{P}(\boldsymbol{\sigma}, \mathcal{H})=\frac{1}{4 \pi} \int_{S_{\zeta}} \frac{\mathcal{H}^{-1} \cdot \boldsymbol{\zeta} \otimes \boldsymbol{\zeta}^{t}{ }^{t} \mathcal{H}^{-1}}{{ }^{t} \mathcal{H}^{-1} \cdot \boldsymbol{\sigma} \cdot \mathcal{H}^{-1} \cdot \boldsymbol{\zeta}} d S_{\zeta}
$$

2 Define a transformed ellipsoid with shape tensor $\mathcal{R}$,

$$
\mathcal{R}=\mathcal{H} \cdot \boldsymbol{\sigma}^{-1 / 2}, \quad \boldsymbol{\sigma}^{-1 / 2}=\sum_{i=1}^{3} \frac{1}{\sqrt{\sigma_{i}}} \underline{E}_{i} \otimes \underline{E}_{i}
$$

with $\sigma_{i}$ the eigenvalue of $\boldsymbol{\sigma}$ and $\underline{E}_{i}$ the associated eigenvector.

$$
\boldsymbol{P}(\boldsymbol{\sigma}, \mathcal{H})=\boldsymbol{\sigma}^{-1 / 2} \cdot \underbrace{\left(\frac{1}{4 \pi} \int_{S_{\zeta}} \frac{\boldsymbol{\mathcal { R }}^{-1} \cdot \boldsymbol{\zeta} \otimes \boldsymbol{\zeta} \cdot{ }^{t} \boldsymbol{\mathcal { R }}^{-1}}{\boldsymbol{\zeta} \cdot{ }^{t} \boldsymbol{\mathcal { R }}^{-1} \cdot \mathcal{R}^{-1} \cdot \boldsymbol{\zeta}} d S_{\zeta}\right)}_{\boldsymbol{P}(\boldsymbol{i}, \mathcal{R})} \cdot \boldsymbol{\sigma}^{-1 / 2}
$$

The anisotropic solution can finally be expressed as

$$
\boldsymbol{P}(\boldsymbol{\sigma}, \mathcal{H})=\boldsymbol{\sigma}^{-1 / 2} . \boldsymbol{P}\left(\boldsymbol{i}, \mathcal{H} \cdot \boldsymbol{\sigma}^{-1 / 2}\right) . \boldsymbol{\sigma}^{-1 / 2}
$$

See $[13,15]$ for details on the numerical calculation. This calculation only needs the solution of elliptic integrals and the algorithm for the eigenvectors and eigenvalues of a symmetric 3-3 matrix. As an example, by using Scientific Computing Tools for Python, available at www.scipy.org, one can use the scipy.special.ellipkinc, scipy.special.ellipeinc, and linalg.eig tools available at www.numpy.org. Note that the transformation of coordinates used to calculate the Hill tensor is already known in geophysics; see [38], which refers to Frank's solution [39].

\subsection{Particular case of aligned spheroidal inclusions with the same symmetry axis as the transversely isotropic matrix}

We detail, as a particular case of the general solution presented in section 6.3, the polarization tensors related to spheroidal inclusions: an oblate spherö̈d $(0<\gamma<1)$ and a prolate spheroïd $(1<\gamma)$, with symmetry axis 3 corresponding to the general equation (49) with $a_{1}=a_{2}=a$ and $a_{3}=a \gamma$. 
One considers a transversely isotropic matrix with conductivity tensor $\boldsymbol{\sigma}_{0}(\underline{n}$ denotes the unit vector along the symmetry axis),

$$
\boldsymbol{\sigma}_{0}=\sigma_{0}\left(\nu^{2} \boldsymbol{i}_{T}+\boldsymbol{i}_{N}\right), \quad \boldsymbol{i}_{N}=\underline{n} \otimes \underline{n}, \quad \boldsymbol{i}_{T}=\boldsymbol{i}-\boldsymbol{i}_{N}
$$

The corresponding transformed ellipsoid $\mathcal{R}=\mathcal{H} . \boldsymbol{\sigma}^{-1 / 2}$ is written as

$$
\mathcal{H}=\boldsymbol{i}_{T}+\gamma \boldsymbol{i}_{N}, \quad{ }^{t} \mathcal{R} . \mathcal{R}=\frac{1}{\sigma_{0}}\left(\frac{1}{\nu^{2}} \boldsymbol{i}_{T}+\gamma^{2} \boldsymbol{i}_{N}\right)
$$

The semi-lengths of the transformed ellipsoid $\left(\underline{n}=\underline{e}_{3}\right)$ are written as

$$
A_{T}=A_{1}=A_{2}=\frac{1}{\nu \sqrt{\sigma_{0}}}, \quad A_{N}=A_{3}=\frac{\gamma}{\sqrt{\sigma_{0}}}
$$

The aspect ratio of the transformed spheroid is defined as

$$
\xi=\frac{A_{N}}{A_{T}}=\nu \gamma
$$

In transversely isotropic rocks, the electrical conductivity tensor generally verifies that $\nu>1$, and the following particular cases may be distinguished:

- oblate solution: $0<\gamma<\frac{1}{\nu}$

- spherical solution: $\gamma=\frac{1}{\nu}$, the considered spheroid is oblate $\left(\gamma=\frac{1}{\nu}<1\right)$ but the transformed spheroid is a sphere

- prolate solution: $\frac{1}{\nu}<\gamma$; it may be noted that if $\frac{1}{\nu}<\gamma<1$, the spheroid is oblate but the transformed spheroid is prolate

The Hill polarisation tensor $\boldsymbol{P}$ of a spheroidal inclusion aligned in the direction of a transversely isotropic matrix (the spheroid and matrix have the same symmetry axis) is written as

$$
\boldsymbol{P}=\frac{I_{T}}{\nu^{2} \sigma_{0}} \boldsymbol{i}_{T}+\frac{I_{N}}{\sigma_{0}} \boldsymbol{i}_{N}, \quad I_{T}=g(\nu \gamma), \quad I_{N}=1-2 g(\nu \gamma)
$$


6.5. Particular case of spheroidal inhomogeneities with symmetry axis perpendicular to the symmetry axis of a transversely isotropic matrix

It is convenient, for this case, to consider the intermediate basis $\underline{e}_{i}^{\varphi_{\alpha}}=$ $Q_{i j}^{\varphi_{\alpha}} \underline{e}_{j}$ detailed in appendix 7. In this basis, as $\underline{e}_{3}^{\varphi_{\alpha}}=\underline{e}_{3}$ (symmetry axis of the matrix), the matrix conductivity tensor is written as

$$
\boldsymbol{\sigma}_{0}=\sigma_{0}\left(\nu^{2}\left(\underline{e}_{1}^{\varphi_{\alpha}} \otimes \underline{\varphi}_{1}^{\varphi_{\alpha}}+\underline{e}_{2}^{\varphi_{\alpha}} \otimes \underline{e}_{2}^{\varphi_{\alpha}}\right)+\underline{e}_{3}^{\varphi_{\alpha}} \otimes \underline{e}_{3}^{\varphi_{\alpha}}\right)
$$

The shape tensor of a spheroid with symmetry axis $\underline{e}_{3}^{\alpha}$ is written as

$$
\mathcal{H}=\underline{e}_{1}^{\alpha} \otimes \underline{e}_{1}^{\alpha}+\underline{e}_{2}^{\alpha} \otimes \underline{e}_{2}^{\alpha}+\gamma \underline{e}_{3}^{\alpha} \otimes \underline{e}_{3}^{\alpha}
$$

A rotation of angle $\theta_{\alpha}=\frac{\pi}{2}$ leads to the following shape tensor in the intermediate basis:

$$
\mathcal{H}=\underline{e}_{1}^{\varphi_{\alpha}} \otimes \underline{e}_{1}^{\varphi_{\alpha}}+\gamma \underline{e}_{2}^{\varphi_{\alpha}} \otimes \underline{e}_{2}^{\varphi_{\alpha}}+\underline{e}_{3}^{\varphi_{\alpha}} \otimes \underline{e}_{3}^{\varphi_{\alpha}}
$$

The corresponding transformed ellipsoid $\mathcal{R}=\mathcal{H} \cdot \boldsymbol{\sigma}^{-1 / 2}$ is diagonal and orthotropic,

$$
{ }^{t} \mathcal{R} . \mathcal{R}=\frac{1}{\sigma_{0}}\left(\frac{1}{\nu^{2}} \underline{e}_{1}^{\varphi_{\alpha}} \otimes \underline{e}_{1}^{\varphi_{\alpha}}+\frac{\gamma^{2}}{\nu^{2}} \underline{e}_{2}^{\varphi_{\alpha}} \otimes \underline{e}_{2}^{\varphi_{\alpha}}+\underline{e}_{3}^{\varphi_{\alpha}} \otimes \underline{e}_{3}^{\varphi_{\alpha}}\right)
$$

The semi-lengths of the transformed ellipsoid are written as

$$
a_{1}^{\varphi_{\alpha}}=\frac{1}{\nu \sqrt{\sigma_{0}}}<a_{3}^{\varphi_{\alpha}}=\frac{1}{\sqrt{\sigma_{0}}}, \quad a_{2}^{\varphi_{\alpha}}=\frac{\gamma}{\nu \sqrt{\sigma_{0}}}
$$

\section{Appendix. Averaged tensors in the transversely isotropic frame}

\subsection{Transformations of coordinates useful in the case of a transversely isotropic matrix}

We present in this section details of the calculus of tensors averaged over orientation in the particular case of a transversely isotropic matrix with embedded spheroidal inclusions. In the case of orientation, the distributions verified the symmetry of the revolution about the same axis as the matrix.The effective conductivity is transversely isotropic with the same symmetry axis as the matrix. An overline denotes averaging over orientation; in the case of a second-rank tensor $\boldsymbol{B}$, this is written as

$$
\overline{\boldsymbol{B}}=\bar{B}_{i j} \underline{e}_{i} \otimes \underline{e}_{j}
$$




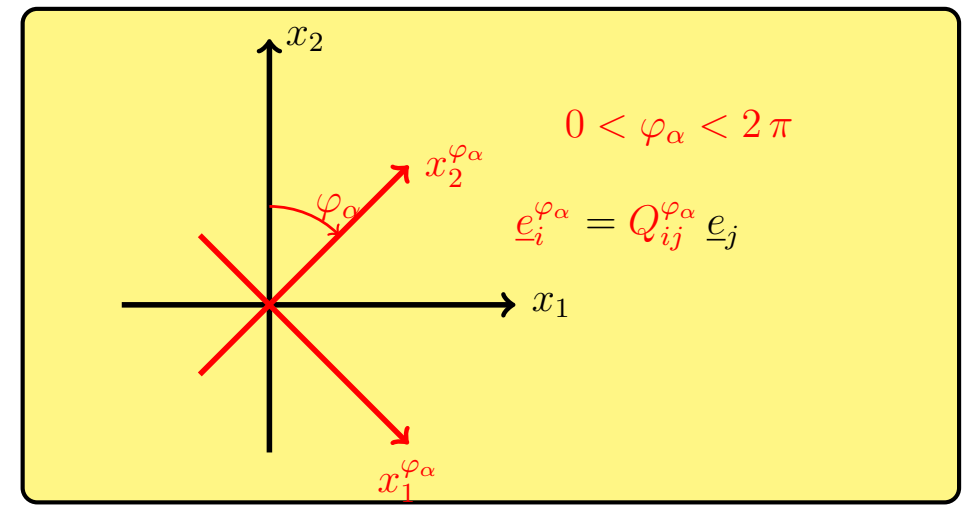

Figure 5: $\varphi_{\alpha}$ angle characterizing rotation in transversely isotropic plane (longitude spherical angle about the symmetry axis of the material)

$$
\bar{B}_{i j}=\frac{1}{4 \pi} \int_{\varphi_{\alpha}=0}^{2 \pi} \int_{\theta_{\alpha}=0}^{\pi} B_{i j}\left(\varphi_{\alpha}, \theta_{\alpha}\right) \sin \theta_{\alpha} d \theta_{\alpha} d \varphi_{\alpha}
$$

where spherical angles $\varphi_{\alpha}$ and $\theta_{\alpha}$ are shown in figures 7.1-7.1 (with the $\underline{e}_{3}$ symmetry axis of the transversely isotropic matrix and material).

$$
\underline{e}_{i}^{\varphi_{\alpha}}=Q_{i j}^{\varphi_{\alpha}} \underline{e}_{j}
$$

The corresponding non-zero components of the transformation matrices are written as

$$
\begin{gathered}
Q_{11}^{\varphi_{\alpha}}=Q_{22}^{\varphi_{\alpha}}=\cos \varphi_{\alpha}, \quad Q_{33}^{\varphi_{\alpha}}=1, \quad Q_{12}^{\varphi_{\alpha}}=-Q_{21}^{\varphi_{\alpha}}=-\sin \varphi_{\alpha} \\
\underline{e}_{i}^{\alpha}=Q_{i j}^{\theta_{\alpha}} \underline{e}_{j}^{\varphi_{\alpha}}=Q_{i j}^{\alpha} \underline{e}_{j} \\
Q_{11}^{\theta_{\alpha}}=1, \quad Q_{22}^{\theta_{\alpha}}=Q_{33}^{\theta_{\alpha}}=\cos \theta_{\alpha}, \quad Q_{23}^{\theta_{\alpha}}=-Q_{32}^{\theta_{\alpha}}=-\sin \theta_{\alpha}
\end{gathered}
$$




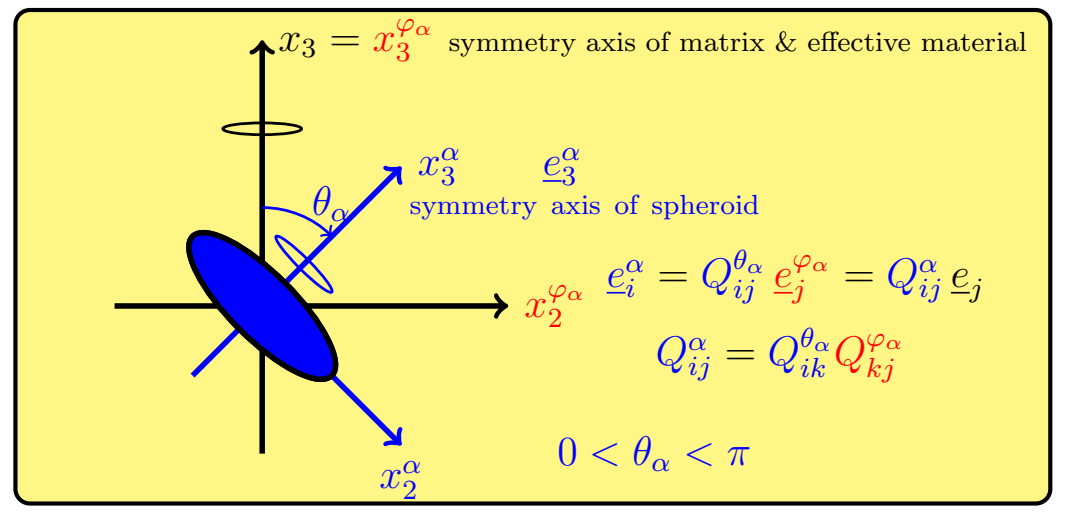

Figure 6: Inclination angle $\theta_{\alpha}$ between the symmetry axes of the matrix and a spheroidal inclusion (colatitude spherical angle about the symmetry axis of the material)

\subsection{Case c: random orientation distribution and numerical integration over} colatitude angle $\theta_{\alpha}$

By using the integration method detailed in [16], one obtains

$$
B_{i j}\left(\varphi_{\alpha}, \theta_{\alpha}\right)=Q_{k i}^{\varphi_{\alpha}}\left(\varphi_{\alpha}\right) Q_{l j}^{\varphi_{\alpha}}\left(\varphi_{\alpha}\right) B_{k l}^{\varphi_{\alpha}}\left(\theta_{\alpha}\right)
$$

The averaged tensor is transversely isotropic,

$$
\begin{gathered}
\bar{B}=\bar{B}_{T} \boldsymbol{i}_{T}+\bar{B}_{N} \boldsymbol{i}_{N} \\
\bar{B}_{T}=\frac{1}{4} \int_{\theta_{\alpha}=0}^{\pi}\left(B_{11}^{\varphi_{\alpha}}\left(\theta_{\alpha}\right)+B_{22}^{\varphi_{\alpha}}\left(\theta_{\alpha}\right)\right) \sin \theta_{\alpha} d \theta_{\alpha} \\
\bar{B}_{N}=\frac{1}{2} \int_{\theta_{\alpha}=0}^{\pi} B_{33}^{\varphi_{\alpha}}\left(\theta_{\alpha}\right) \sin \theta_{\alpha} d \theta_{\alpha}
\end{gathered}
$$

The Gaussian quadrature rule is finally used to calculate integrals (93-94)

\subsection{Case b: axially random orientation distribution}

This case is simpler than the previous case, as we consider a constant angle $\varphi_{\alpha}=\frac{\pi}{2}$ (see figure 7.3). For Hill, the conductivity contribution and concentration tensor of isolated inhomogeneities are orthotropic. A random 


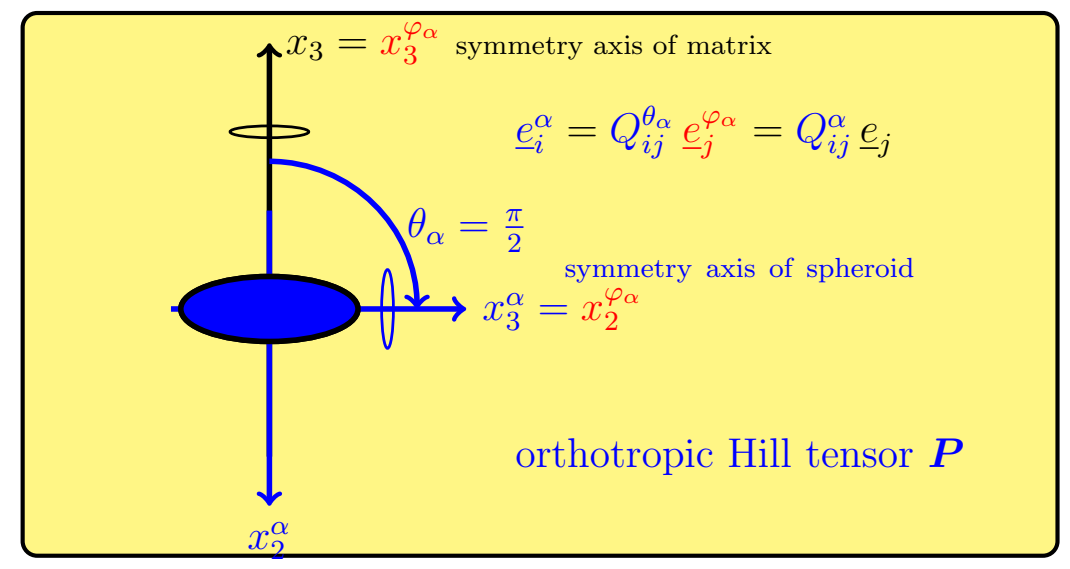

Figure 7: Inclination angle $\theta_{\alpha}=\pi / 2$ between the symmetry axes of the matrix and a spheroidal inclusion

orientation distribution in the transversely isotropic plane conduces to transversely isotropic averaged tensors with the same symmetry axis as the matrix. By using the integration method detailed in [16], one obtains

$$
B_{i j}\left(\varphi_{\alpha}\right)=Q_{k i}^{\varphi_{\alpha}}\left(\varphi_{\alpha}\right) Q_{l j}^{\varphi_{\alpha}}\left(\varphi_{\alpha}\right) B_{k l}^{\varphi_{\alpha}}
$$

The averaged tensor is transversely isotropic,

$$
\begin{gathered}
\bar{B}_{i j}=\frac{1}{2 \pi} \int_{\varphi_{\alpha}=0}^{2 \pi} B_{i j}\left(\varphi_{\alpha}\right) d \varphi_{\alpha}=\frac{1}{2 \pi} \int_{\varphi_{\alpha}=0}^{2 \pi} Q_{k i}^{\varphi_{\alpha}}\left(\varphi_{\alpha}\right) Q_{l j}^{\varphi_{\alpha}}\left(\varphi_{\alpha}\right) B_{k l}^{\varphi_{\alpha}} d \varphi_{\alpha} \\
\bar{B}=\bar{B}_{T} \boldsymbol{i}_{T}+\bar{B}_{N} \boldsymbol{i}_{N} \\
\bar{B}_{T}=\frac{B_{11}^{\varphi_{\alpha}}+B_{22}^{\varphi_{\alpha}}}{2}, \quad \bar{B}_{N}=B_{33}^{\varphi_{\alpha}}
\end{gathered}
$$

\section{Acknowledgement}

$A G$ gratefully acknowledges the financial support from the Scientific Association for Geology and its Applications ASGA, Nancy, France. $P C$ and $D P$ gratefully acknowledge the support from the $E x C i T I N G$ research project funded by the French National Research Agency (grant agreement ANR-17CE06-0012). 


\section{References}

[1] J. Sarout, L. Molez, Y. Guéguen, N. Hoteit, Shale dynamic properties and anisotropy under triaxial loading: Experimental and theoretical investigations, Physics and Chemistry of the Earth 32 (2007) 896-906.

[2] C. Bobko, F. Ulm, The nano-mechanical morphology of shale, Mechanics of Materials 40 (2008) 318-337.

[3] I. O. Bayuk, M. Ammerman, E. M. Chesnokov, Upscaling of elastic properties of anisotropic sedimentary rocks, Geophysical Journal International 172 (2) (2008) 842-860.

[4] P. Cosenza, D. Prêt, A. Giraud, S. Hedan, Effect of the local clay distribution on the effective elastic properties of shales, Mechanics of Materials 84 (2015) $55-74$.

[5] J.-C. Robinet, P. Sardini, D. Coelho, J.-C. Parneix, D. Prêt, S. Sammartino, E. Boller, S. Altmann, Effects of mineral distribution at mesoscopic scale on solute diffusion in a clay-rich rock: Example of the Callovo-Oxfordian mudstone (Bure, France), Water Resources Research $48(5)$.

[6] P. Cosenza, D. Prêt, M. Zamora, Effect of the local clay distribution on the effective electrical conductivity of clay rocks, Journal of Geophysical Research: Solid Earth 120 (1) (2015) 145-168.

[7] L. M. Keller, P. Schuetz, R. Erni, M. D. Rossell, F. Lucas, P. Gasser, L. Holzer, Characterization of multi-scale microstructural features in Opalinus Clay, Microporous and Mesoporous Materials 170 (2013) 83 94.

[8] A. Revil, Transport of water and ions in partially water-saturated porous media. Part 1. Constitutive equations, Advances in Water Resources 103 (2017) $119-138$.

[9] A. Revil, Transport of water and ions in partially water-saturated porous media. Part 2. Filtration effects, Advances in Water Resources 103 (2017) $139-152$. 
[10] A. Revil, A. S. Ahmed, S. Matthai, Transport of water and ions in partially water-saturated porous media. Part 3. Electrical conductivity, Advances in Water Resources 121 (2018) 97 - 111.

[11] V. T. Ebufegha, Anisotropy of Mudrocks: Quantifying Controls and Fabric Implications in the Horn River Basin, Ph.D. thesis, Department of Physics, University of Alberta (2016).

[12] L. Comparon, Etude expérimentale des propriétés et diélectriques des matériaux argileux consolidés (in french), Ph.D. thesis, Institut de Physique du Globe de Paris (2005).

[13] J. F. Barthélémy, Effective permeability of media with a dense network of long and micro fractures, Transport in Porous Media 76 (1) (2008) $153-178$.

[14] S. Giordano, P. L. Palla, Dielectric behavior of anisotropic inhomogeneities: interior and exterior point eshelby tensors, Journal of Physics A: Mathematical and Theoretical 41 (41) (2008) 415205.

[15] P. N. Sævik, I. Berre, M. Jakobsen, M. Lien, A 3D computational study of effective medium methods applied to fractured media, Transport in Porous Media 100 (1) (2013) 115-142.

[16] A. Giraud, C. Gruescu, D. P. Do, F. Homand, D. Kondo, Effective thermal conductivity of transversely isotropic media with arbitrary oriented ellipsordal inhomogeneities, International Journal of Solids and Structures 44 (11) (2007) 2627-2647.

[17] A. Giraud, Q. V. Huynh, D. Hoxha, D. Kondo, Effective poroelastic properties of transversely isotropic rocks-like composites with arbitrarily oriented ellipsoidal inclusions, Mechanics of Materials 39 (11) (2007) 1006-1024.

[18] M. Hori, S. Nemat-Nasser, Overall moduli of solids with microcracks: Load induced anisotropy, Journal of the Mechanics and Physics of Solids 31 (2) (1983) 155-171.

[19] I. Sevostianov, M. Kachanov, Explicit cross-property correlations for anisotropic two-phase composite materials, Journal of the Mechanics and Physics of Solids 50 (2) (2002) $253-282$. 
[20] A. Trofimov, S. Abaimov, I. Sevostianov, Inverse homogenization problem: Evaluation of elastic and electrical (thermal) properties of composite constituents, International Journal of Engineering Science 129 (2018) $34-46$.

[21] T. Mori, K. Tanaka, Average stress in matrix and average elastic energy of materials with misfitting inclusions, Acta Metall. 21 (1973) 571-574.

[22] Y. Benveniste, A new approach to the application of Mori-Tanaka's theory in composite materials, Mechanics of Materials 6 (2) (1987) 147 $-157$.

[23] T. Mishurova, N. Rachmatulin, P. Fontana, T. Oesch, G. Bruno, E. Radi, I. Sevostianov, Evaluation of the probability density of inhomogeneous fiber orientations by computed tomography and its application to the calculation of the effective properties of a fiber-reinforced composite, International Journal of Engineering Science 122 (Supplement C) (2018) $14-29$.

[24] I. Sevostianov, M. Kachanov, Effective Properties of Heterogeneous Materials, I. Sevostianov and M. Kachanov Edition, Springer Netherlands, 2013, Ch. Non Interaction Approximation in the Problem of Effective Properties, pp. 1-96.

[25] V. Kushch, I. Sevostianov, Maxwell homogenization scheme as a rigorous method of micromechanics: Application to effective conductivity of a composite with spheroidal particles, International Journal of Engineering Science 98 (2016) 36 - 50.

[26] I. Sevostianov, On the shape of effective inclusion in the Maxwell homogenization scheme for anisotropic elastic composites, Mechanics of Materials 75 (0) (2014) $45-59$.

[27] P. Ponte Castañeda, J. R. Willis, The effect of spatial distribution on the effective behavior of composite materials and cracked media, Journal of the Mechanics and Physics of Solids 43 (12) (1995) 1919-1951.

[28] S. Giordano, Nonlinear effective properties of heterogeneous materials with ellipsoidal microstructure, Mechanics of Materials 105 (2017) 16 28. 
[29] S. Torquato, Random Heterogenous Materials. Microstructure and Macroscopic Properties, Springer-Verlag, 2002.

[30] M. Markov, A. Mousatov, E. Kazatchenko, I. Markova, Determination of electrical conductivity of double-porosity formations by using generalized differential effective medium approximation, Journal of Applied Geophysics 108 (0) (2014) $104-109$.

[31] A. AbouChakraGuéry, F. Cormery, J.-F. Shao, D. Kondo, A comparative micromechanical analysis of the effective properties of a geomaterial: Effect of mineralogical compositions, Computers and Geotechnics 37 (5) (2010) $585-593$.

[32] S. Nguyen, M.-H. Vu, M. Vu, Extended analytical approach for electrical anisotropy of geomaterials, Journal of Applied Geophysics 123 (2015) $211-217$.

[33] A. N. Norris, A differential scheme for the effective moduli of composites, Mechanics of Materials 4 (1) (1985) 1 - 16.

[34] V. I. Kushch, Interacting ellipsoidal inhomogeneities by multipole expansion method and effective conductivity of particulate composite, International Journal of Engineering Science 115 (2017) 1 - 13.

[35] V. I. Kushch, I. Sevostianov, A. Giraud, Local fields and effective conductivity tensor of ellipsoidal particle composite with anisotropic constituents, Proceedings of the Royal Society A. 473 (1) (2017) $53-77$.

[36] J. F. Barthélémy, Homogénéisation de la perméabilité d'un milieu fracturé anisotrope et rôle de la perméabilité transverse des fractures, in: Congrès Français de Mécanique, Grenoble, France, 2007.

[37] T. Mura, Micromechanics of defects in solids, 2nd Edition, Martinus Nijhoff Publishers, 1987.

[38] K. S. Mendelson, M. H. Cohen, The effect of grain anisotropy on the electrical properties of sedimentary rocks, Geophysics 47 (2) (1982) 257 $-263$.

[39] V. Frank, On the penetration of a static homogeneous field in an anisotropic medium into an ellipsoidal inclusion consisting of another 
anisotropic medium, in: Electromagnetic theory and antennas: E. C. Jordan, The Macmillan Co, New York, 1963, p. 615. 


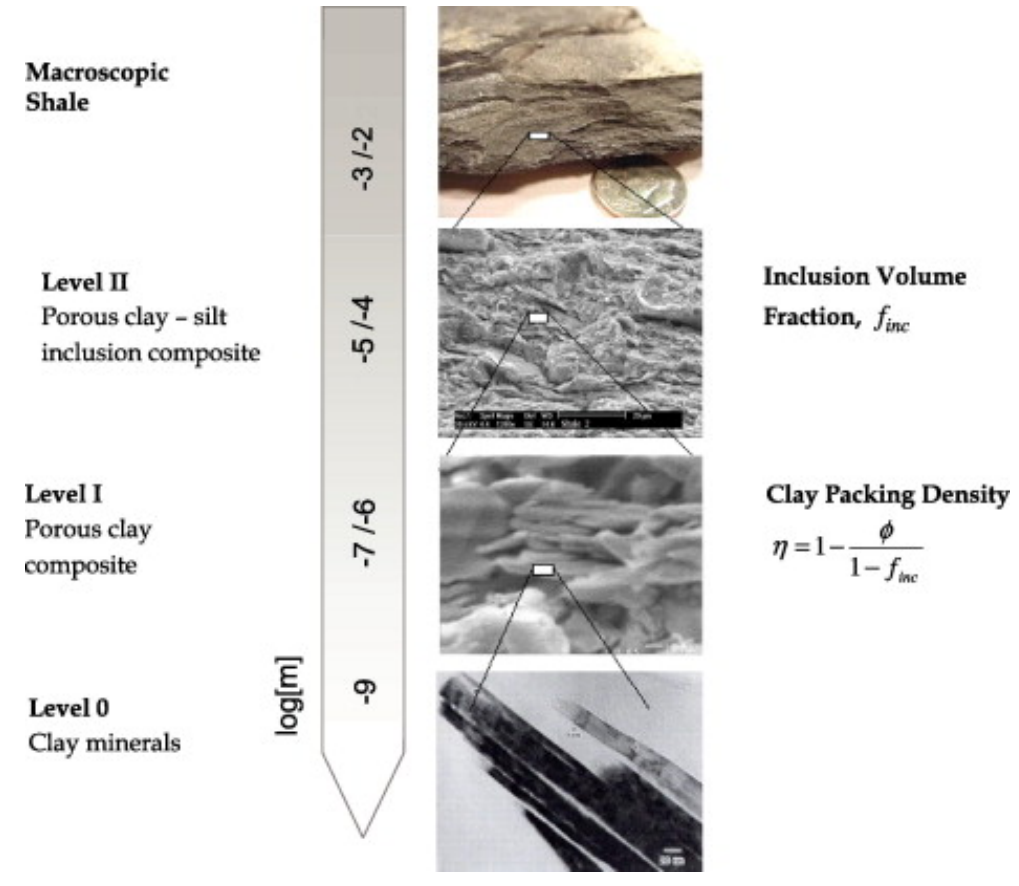

Figure 8: Multiscale model for shale, figure from [2]. The mesoscale considered in this paper corresponds to level $I I$ and the macroscale to level $I I I$

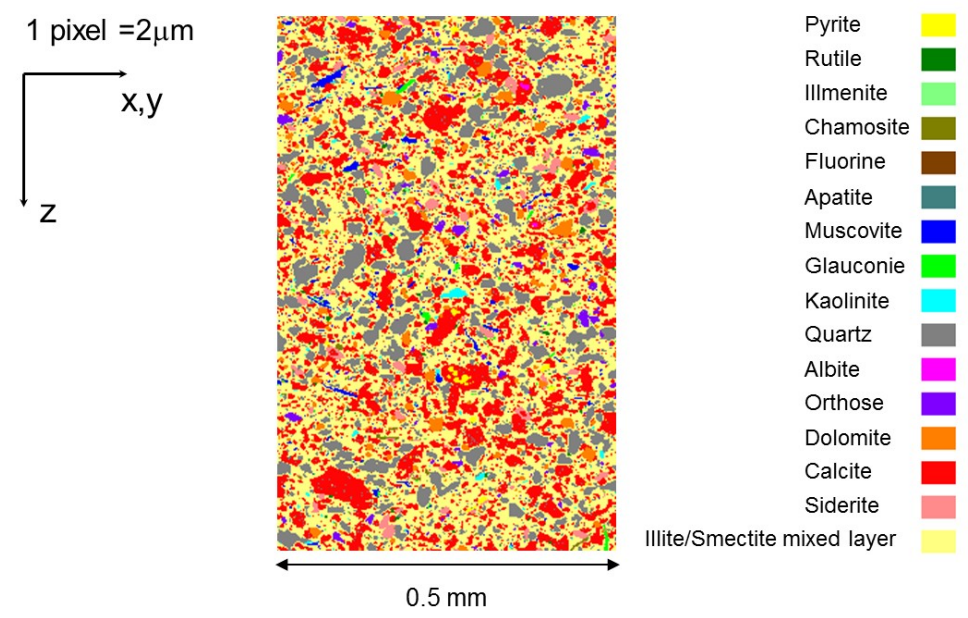

Figure 9: Mineral map of a sample of Callovo-Oxfordian mudstone [6] 


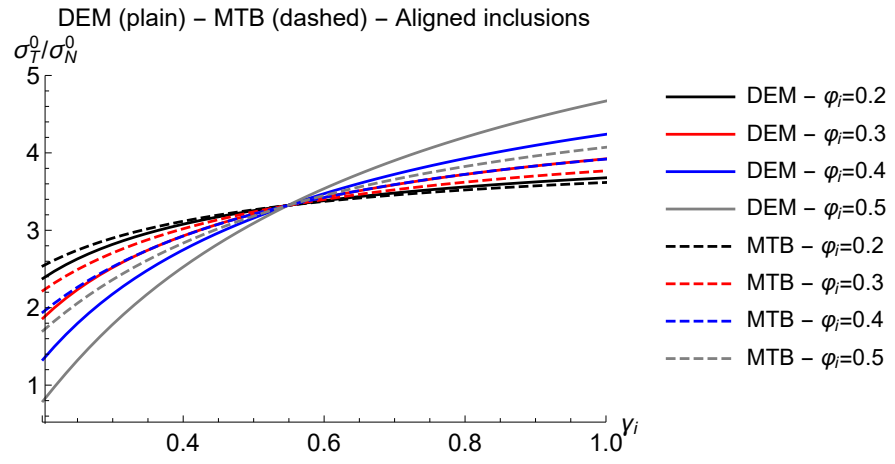

Figure 10: Case a: aligned distribution of inclusions. Ratio of the transverse to normal matrix conductivity as a function of the aspect ratio $\gamma_{i}$ of inclusions (only the oblate case) for different values of the volume fraction $\varphi_{i}$ of the inclusion phase.

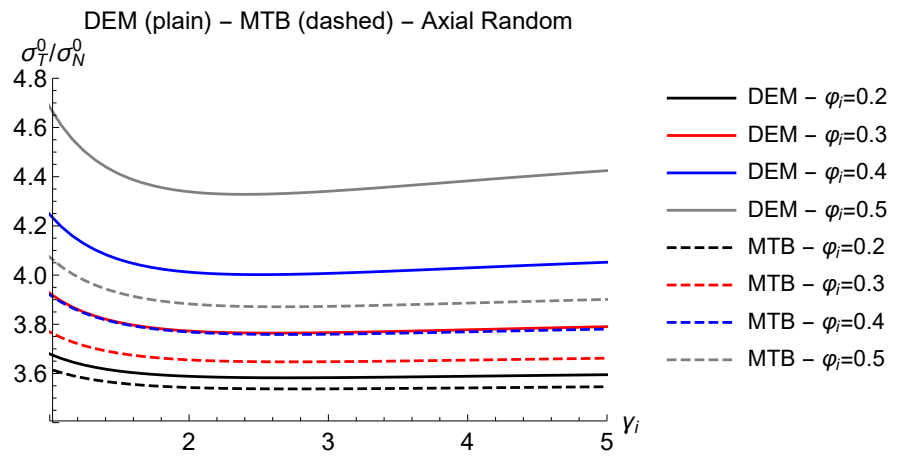

Figure 11: Case b: inclusions randomly distributed in the transversely isotropic plane (axially random distribution). Ratio of the transverse to normal matrix conductivity as a function of $\gamma_{i}$ (oblate and prolate cases) for different values of the volume fraction $\varphi_{i}$ of the inclusion phase. 


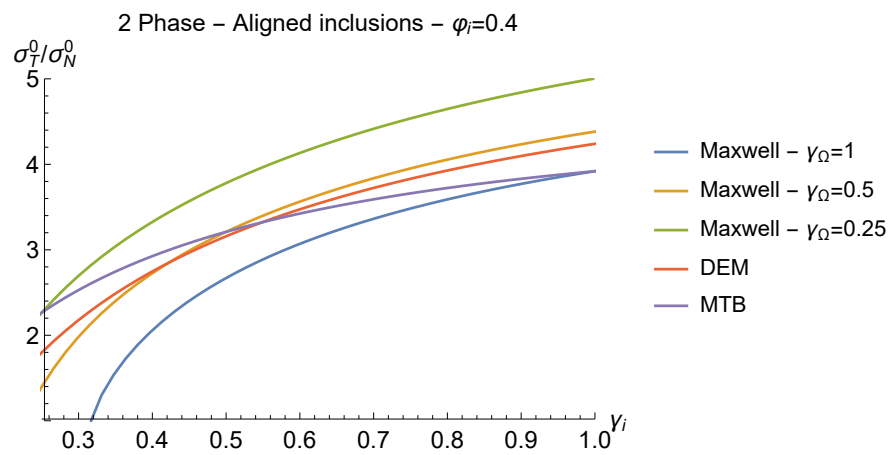

Figure 12: Case a: aligned distribution of inclusions. Ratio of the transverse to normal matrix conductivity as a function of $\gamma_{i}$ for teh DEM, MTB and Maxwell homogenization schemes with different aspect ratios of the effective spheroidal inclusion.

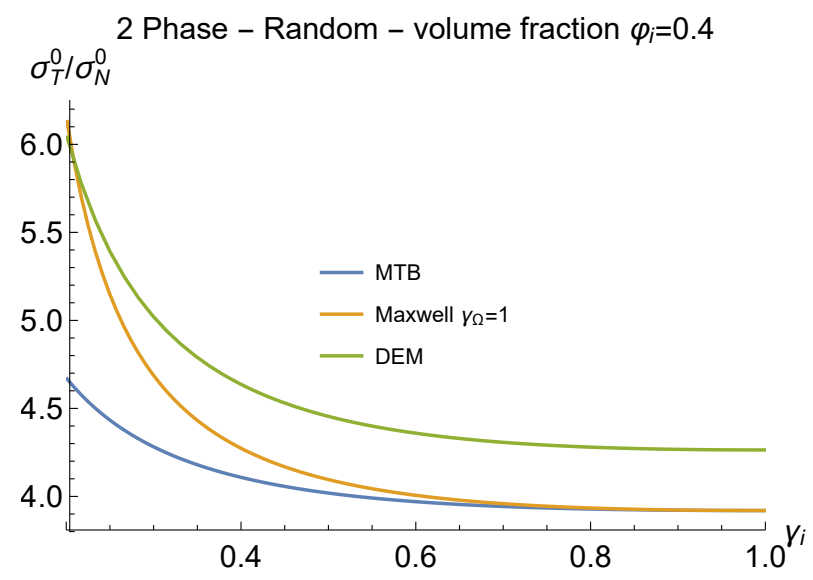

Figure 13: Case c: random orientation distribution of inclusions. Ratio fo the transverse to normal matrix conductivity as a function of $\gamma_{i}$ for the DEM, MTB and Maxwell homogenization schemes. 


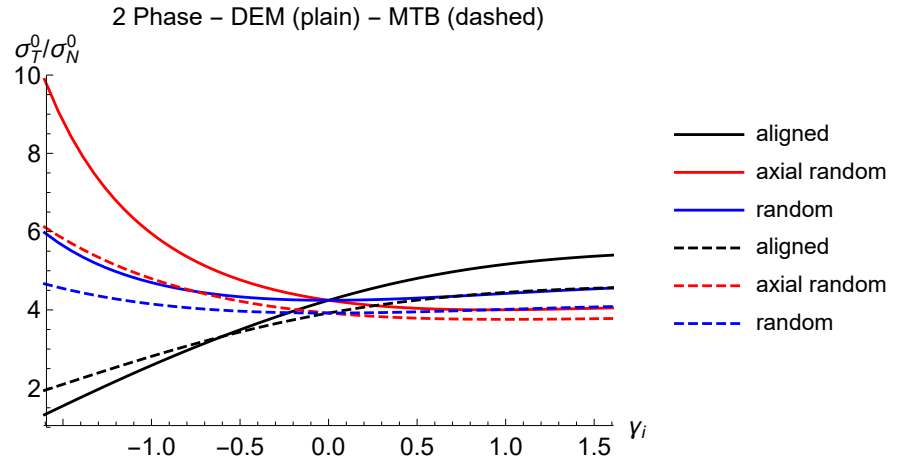

Figure 14: Two-phase model. Comparison between cases a (aligned), b (axially random) \& c (random). Ratio of the transverse to normal matrix conductivity as a function of $\gamma_{i}$
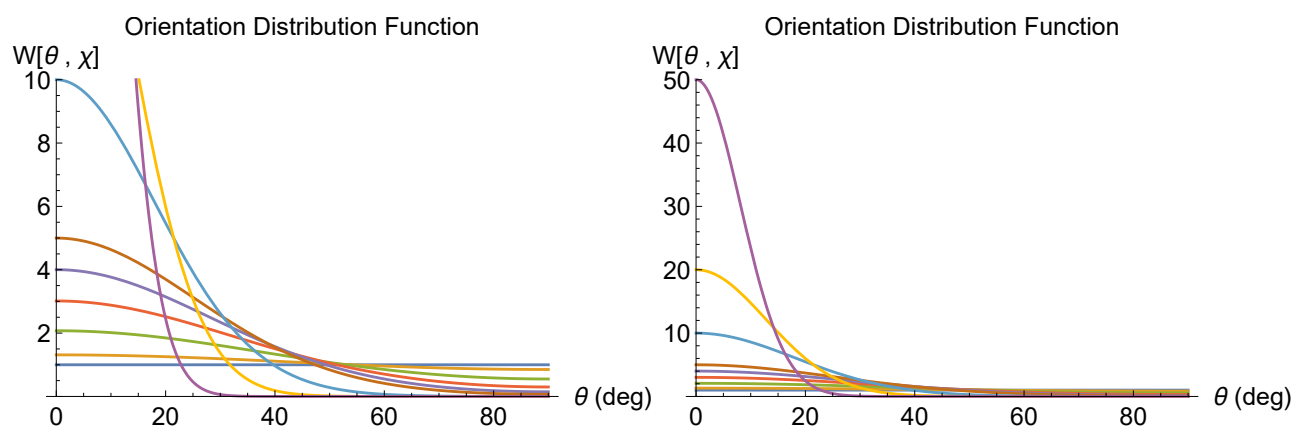

Figure 15: Orientation distribution function $(O D F)$ with argument colatitude angle $\theta_{\alpha}$ and parameter $\chi=0$ (random); $1 ; 2 ; 3 ; 4 ; 5 ; 10 ; 20 ; 50$
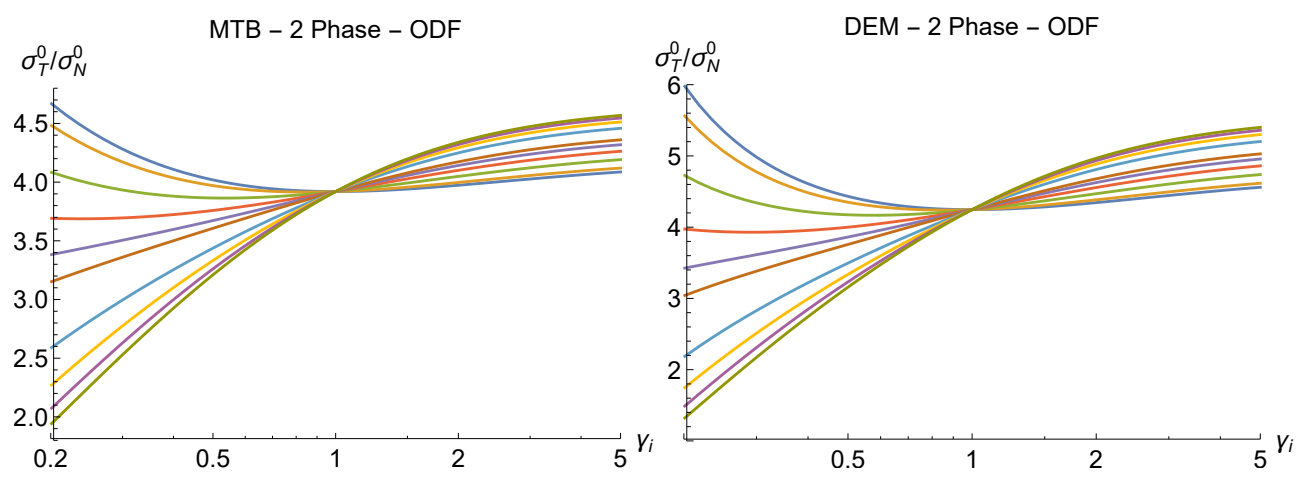

Figure 16: Two-phase model (case d): $O D F$. Ratio of the transverse to normal matrix conductivity as a function of $\gamma_{i}$ for different degrees of alignment (from top to bottom on the left (oblate) $\chi=0$ (random); $1 ; 2 ; 3 ; 4 ; 5 ; 10 ; 20 ; 50$, aligned) 


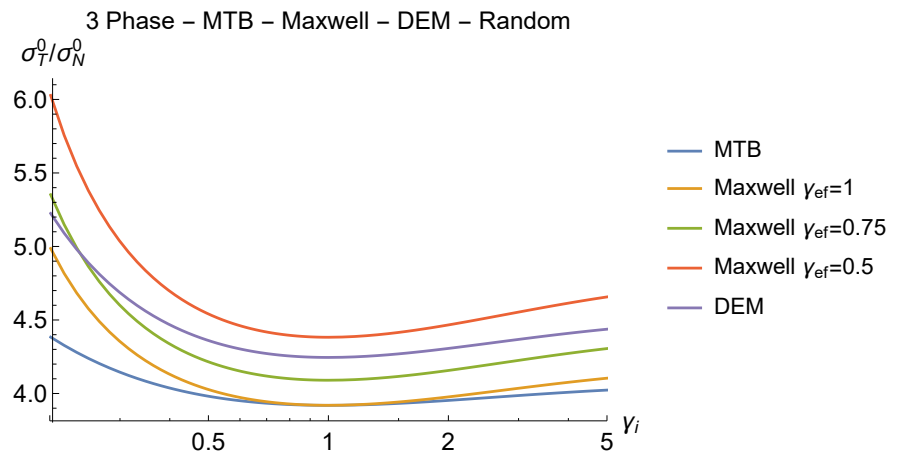

Figure 17: Reference 3-phase model. Random orientation distribution. Ratio of the transverse to normal matrix conductivity as a function of the aspect ratio $\gamma_{1}$ of calcite spheroidal inclusions

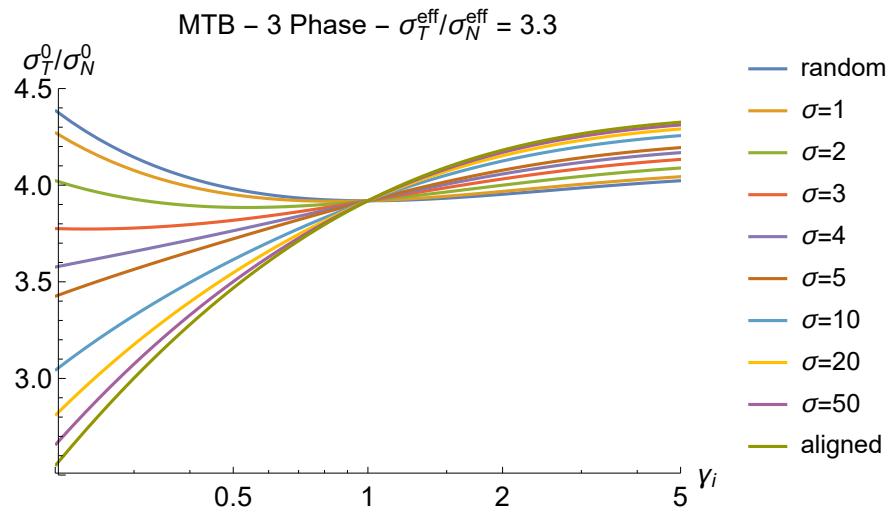

Figure 18: Reference 3-phase model: $O D F$. Ratio of the transverse to normal matrix conductivity as a function of the aspect ratio $\gamma_{1}$ of calcite spheroidal inclusions for different degrees of alignment 


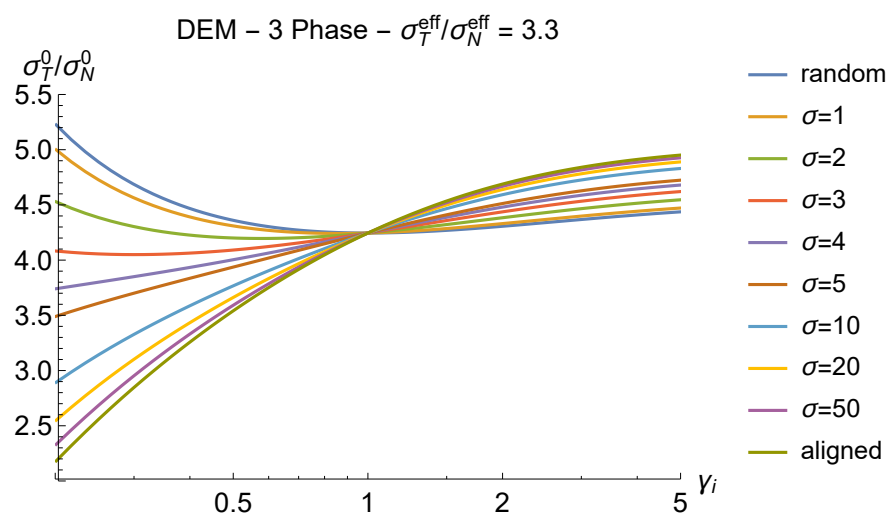

Figure 19: Reference 3-phase model: $O D F$. Ratio of the transverse to normal matrix conductivity as a function of the aspect ratio $\gamma_{1}$ of calcite spheroidal inclusions for different degrees of alignment
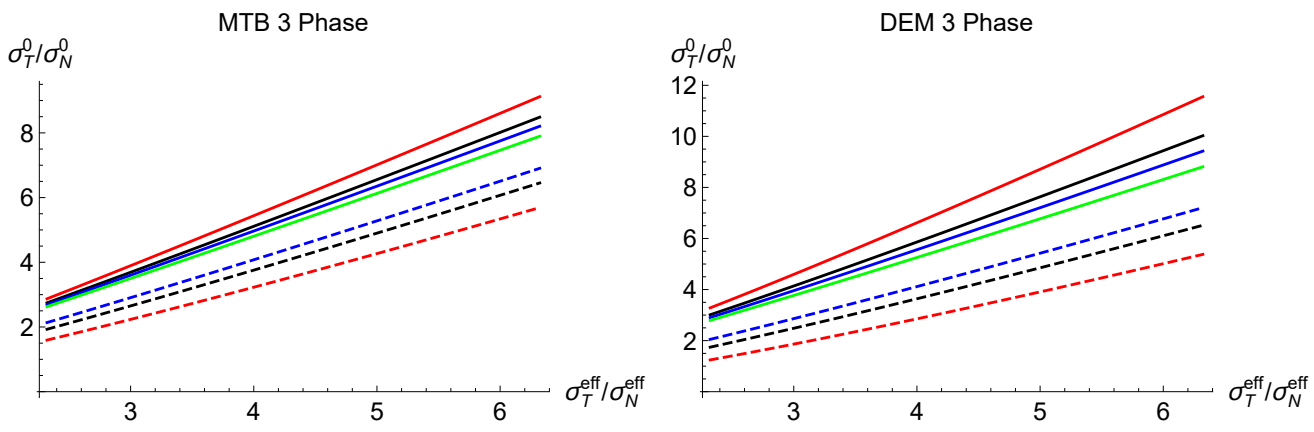

Figure 20: 3-phase model with $2.3<\nu_{\text {eff }}^{2}=\sigma_{T}^{\text {eff }} / \sigma_{N}^{\text {eff }}<6.3$ (plain line: random; dashed line: aligned orientation distributions). Ratio of the transverse to normal matrix conductivity as a function of $\nu_{\text {eff }}^{2}$ for different aspect ratios of calcite inclusions: Red: $\gamma_{1}=0.2$, Black: $\gamma_{1}=0.3$, Blue: $\gamma_{1}=0.4$, Green: $\gamma_{1}=1$. 

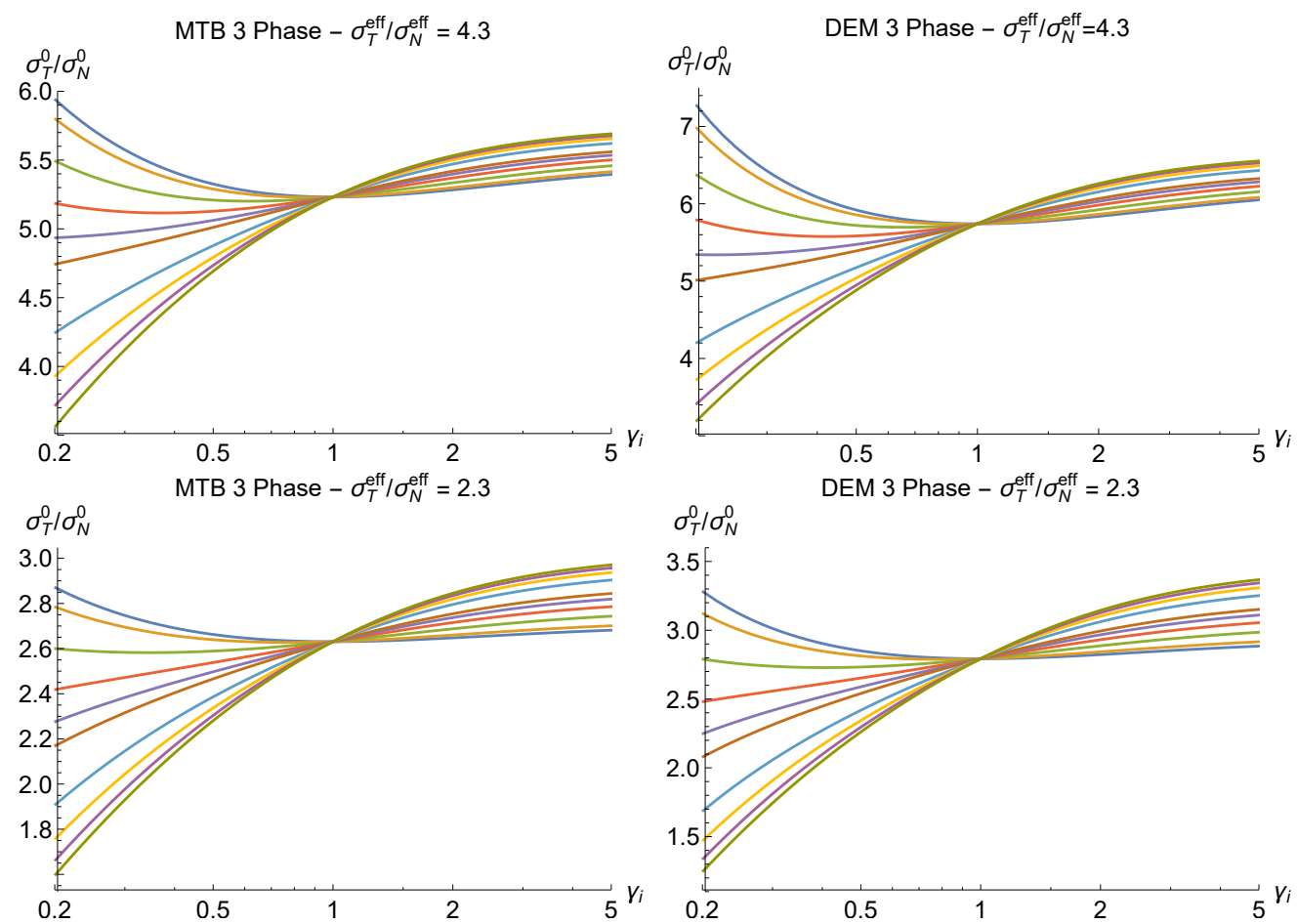

Figure 21: 3-phase model with $\nu_{\text {eff }}^{2}=\sigma_{T}^{\text {eff }} / \sigma_{N}^{\text {eff }}=4.3$ (top) -2.3 (bottom). Ratio of the transverse to normal matrix conductivity as a function of $\gamma_{1}$ for different degrees of alignment (from top to bottom on the left (oblate) $\chi=0$ (random); $; ; 2 ; 3 ; 4 ; 5 ; 10 ; 20 ; 50$, aligned) 

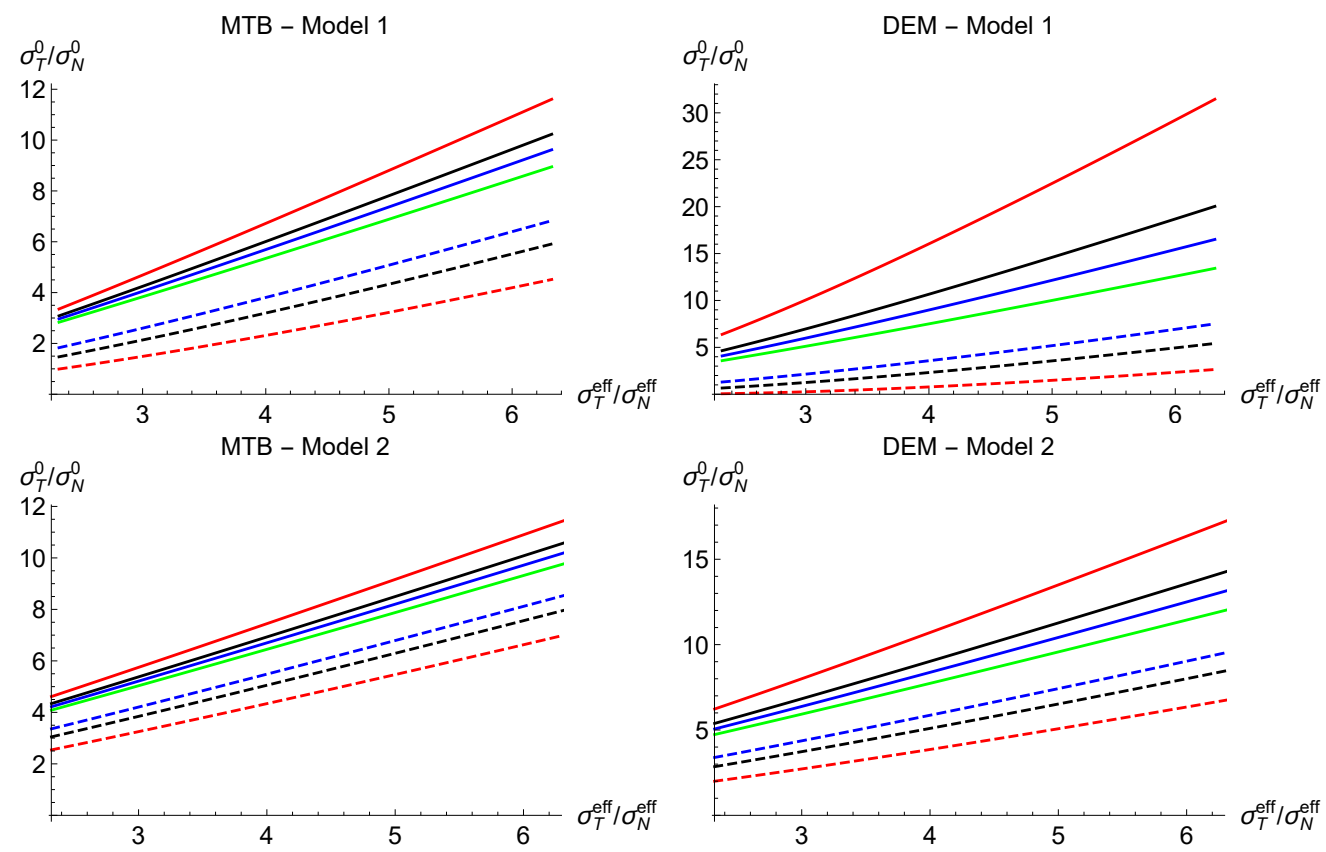

Figure 22: 3-phase model with $2.3<\nu_{\text {eff }}^{2}=\sigma_{T}^{\text {eff }} / \sigma_{N}^{\text {eff }}<6.3$, (plain line: random; dashed line: aligned orientation distributions). Ratio of the transverse to normal matrix conductivity as a function of $\nu_{\text {eff }}^{2}$ for different aspect ratios: Red: $\gamma_{1}=0.2$, Black: $\gamma_{1}=0.3$, Blue: $\gamma_{1}=0.4$, Green: $\gamma_{1}=1$. 

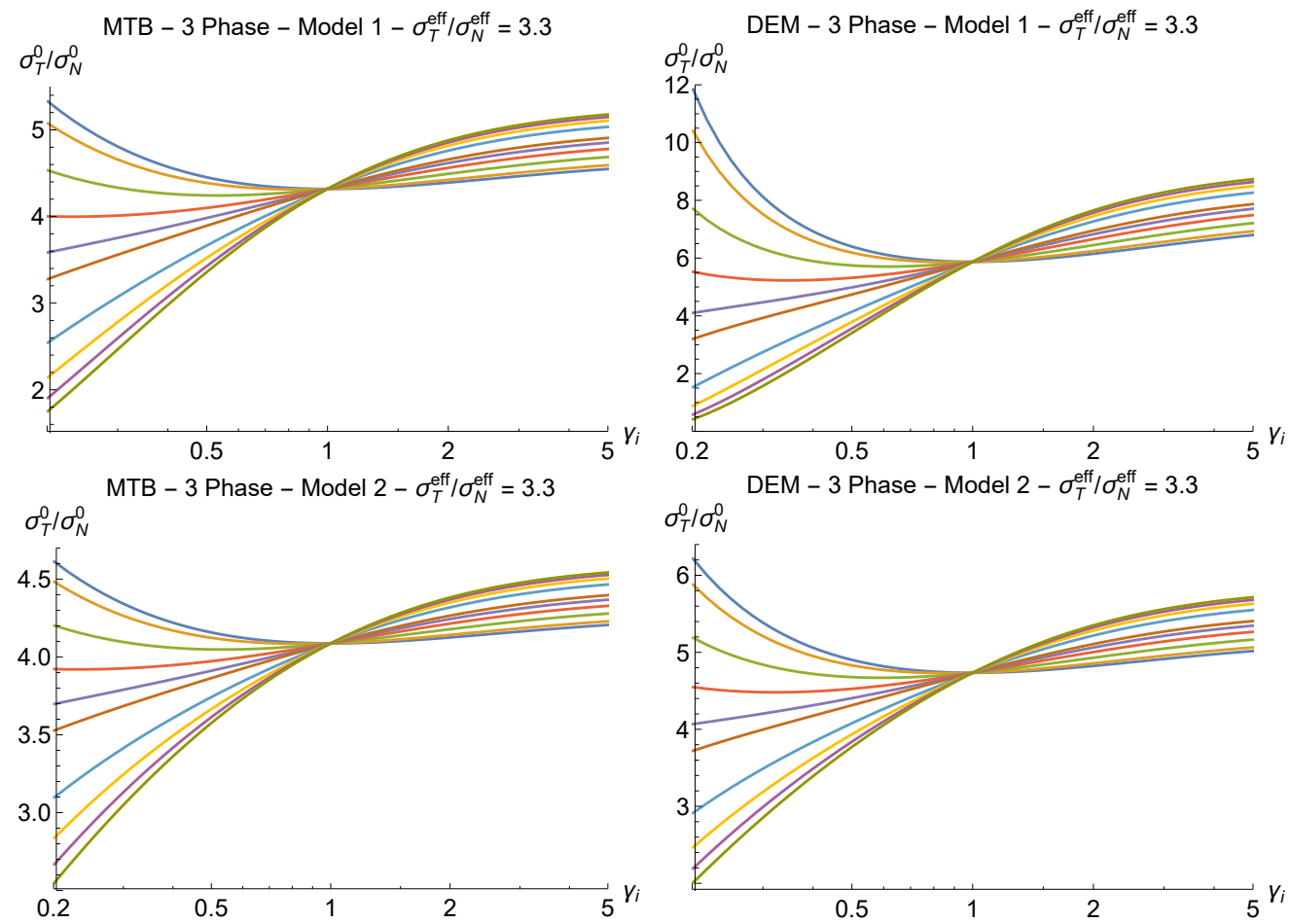

Figure 23: 3-phase model with $\nu_{\text {eff }}^{2}=\sigma_{T}^{\text {eff }} / \sigma_{N}^{\text {eff }}=3.3$ : model 1 (top) and model 2 (bottom). Ratio of the transverse to normal matrix conductivity as a function of $\gamma_{1}$ for different degrees of alignment (from top to bottom on the left (oblate) $\chi=$ 0 (random) $; 1 ; 2 ; 3 ; 4 ; 5 ; 10 ; 20 ; 50$, aligned) 